\title{
Yukawa couplings for intersecting D-branes on non-factorisable tori
}

\author{
Stefan Förste and Christoph Liyanage \\ Bethe Center for Theoretical Physics and Physikalisches Institut der Universität Bonn, \\ Nussallee 12, 53115 Bonn, Germany \\ E-mail: forste@th.physik.uni-bonn.de, liyanage@th.physik.uni-bonn.de
}

\begin{abstract}
We compute Yukawa couplings in type IIa string theory compactified on a sixtorus in the presence of intersecting D6-branes. The six-torus is generated by an $\mathrm{SO}(12)$ root lattice. Yukawa couplings are expressed as sums over worldsheet instantons. Our result extends known expressions to a non-factorisable torus. As an aside we also fill in some details for the factorisable torus and non-coprime intersection numbers.
\end{abstract}

KEYWORDS: Intersecting branes models, D-branes

ArXiv EPRINT: 1412.3645v2 


\section{Contents}

1 Introduction $\quad 1$

2 Intersecting D6-branes and worldsheet instantons 2

3 Branes on non-factorisable six-tori 4

$\begin{array}{lll}4 & \text { Labelling inequivalent intersections } & 7\end{array}$

5 Yukawa couplings $\quad 12$

$\begin{array}{llr}6 & \text { Examples } & 18\end{array}$

$\begin{array}{lll}7 & \text { Conclusions } & 27\end{array}$

A $T^{2}$ with non-coprime intersection numbers $\quad 28$

\section{Introduction}

One of the avenues taken to find an embedding of Standard Model particle physics into string theory consists of intersecting D6-branes in type IIA theory [1-25]. For reviews and a book providing also more references see e.g. [26-29]. Toroidal orientifolds form a small subclass of more general Calabi-Yau compactifications. Apart from their mathematical simplicity the major advantage of toroidal models is that string theory can be exactly quantised in those backgrounds. Various low energy quantities can be computed from scratch. Effective degrees of freedom are explicitly given in terms of string vibration modes. Coupling constants are related to correlation functions of corresponding vertex operators. Interactions in intersecting brane models have been considered in [30-46].

In the present paper we focus on worldsheet instanton contributions to cubic Yukawa couplings. These have been worked out for a factorisable six-torus in [31]. In our context, a factorisable six-torus is a six dimensional torus which can be decomposed into the direct product of three mutually orthogonal two-tori. Yukawa couplings can be written as product of three theta functions where each function's arguments depend on the Kähler modulus of the corresponding two-torus and open string moduli. The authors of [31] speculate that a more general (non-factorisable) six-torus will lead to multi-theta functions. We will show this to be indeed the case by working out details for a six-torus which is the quotient of $\mathbb{R}^{6}$ with respect to shifts by $\mathrm{SO}(12)$ root vectors. Our analysis can be easily extended to other non-factorisable six-tori. Intersecting D-branes on non-factorisable tori and their orientifolds have been discussed in [47-51]. 
In section 2 we recall general statements on how to obtain Yukawa couplings for intersecting D6-branes. Section 3 discusses how to represent cycles wrapped by D6-branes. Here we follow and supplement the presentation of $[48,50]$. A D6-brane is, as in the factorisable case, described by three pairs of wrapping numbers. The closeness to the factorisable representation turns out to be of great advantage in the computation of Yukawa couplings. It is also useful for constructing orientifolds where the point group leaves also factorisable $T^{6}$ invariant. In other cases, such as the $\mathbb{Z}_{12}$ orientifold, a different representation of D6-branes has been employed $[47,51]$. It will be interesting to find out whether our way of characterising the wrapped three-cycle can be useful also for those constructions. Section 4 is devoted on how to label inequivalent intersection points. It turns out that now such inequivalent labels take values on a three dimensional lattice quotiented by a sublattice. In section 5 we derive a general expression for the Yukawa coupling. It is expressed as a sum over a three dimensional lattice resulting in a multi-theta function. In section 6 we illustrate our general procedure at two examples. Section 7 provides our conclusions. In an appendix we revisit a discussion carried out in [31]. We give a more detailed derivation of their result for the case that intersection numbers are not coprime.

\section{Intersecting D6-branes and worldsheet instantons}

Yukawa couplings within intersecting D6-brane models of type IIA string theory have been discussed in general in [31]. There, for type IIA strings compactified on a factorisable six-torus, an explicit computation is presented. The results can be easily extended to the, phenomenologically more relevant, orientifolds of such tori by inclusion of respective image branes. In our context, a factorisable six-torus is a torus which can be decomposed into a product of three mutually orthogonal two-tori. We are going to extend the results of [31] to the case of non-factorisable six-tori. Before doing so, let us recapitulate some general considerations from [31].

After compactifing six of the ten initial spacetime dimensions on a Calabi-Yau threefold $\mathcal{M}=C Y^{3}$, the whole non-compact Minkowski space $\mathbb{M}^{4}$ will be filled with D6-branes. In compact space they will wrap special Lagrangian 3-cycles $\Pi_{a} \in H_{3}(\mathcal{M}, \mathbb{Z})$ :

$$
\mathbb{M}^{4} \times \Pi_{a} \subset \mathbb{M}^{4} \times \mathcal{M} .
$$

Compactification of type II theories then leads to $\mathcal{N}=2$ supersymmetry in four dimensions. Breaking to the phenomenologically interesting amount of $\mathcal{N}=1$ supersymmetry will be discussed shortly. Calabi-Yau threefolds are Ricci-flat Kähler manifolds $\mathcal{M}$ with a complex structure $J$, a Riemannian metric $g$ and additionally a nowhere vanishing holomorphic (3,0)-form $\Omega$ called the volume form satisfying

$$
\frac{\omega^{3}}{3 !}=-\left(\frac{i}{2}\right)^{3} \Omega \wedge \bar{\Omega},
$$

where $\omega$ is the Kähler 2-form of $g$ and $J$. Both, the volume form $\Omega$ and the Kähler 2-form $\omega$, are used to construct calibration forms on $C Y^{3}$. For the 3 -cycles $\Pi_{a}$ to become special Lagrangian, they must be calibrated by a real closed three form with proper normalisation, 
viz. $\cos \theta \operatorname{Re} \Omega+\sin \theta \operatorname{Im} \Omega=\operatorname{Re}\left(\mathrm{e}^{-\mathrm{i} \theta} \Omega\right.$ ) (see e.g. [52]). A calibrated submanifold is a respresentative of a given homology class with minimal volume. For Calabi-Yau manifolds an equivalent condition for submanifolds calibrated w.r.t. $\operatorname{Re}\left(\mathrm{e}^{-\mathrm{i} \theta} \Omega\right)$ is [53]

$$
\left.\omega\right|_{\Pi_{a}} \equiv 0 \quad \text { and }\left.\quad \operatorname{Im}\left(e^{-i \theta} \Omega\right)\right|_{\Pi_{a}} \equiv 0 .
$$

The factor of $e^{-i \theta}$ is a phase, where $\theta$ parameterises how a $\mathrm{U}(1)_{R}$ symmetry of an $\mathcal{N}=1$ supersymmetry is embedded into the $\mathrm{SU}(2)_{R}$ of the, so far, unbroken $\mathcal{N}=2$ superalgebra. Indeed, placing a D-brane on a special Lagrangain cycle breaks $\mathcal{N}=2$ to the corresponding $\mathcal{N}=1$ supersymmetry [54].

Due to Gauss' law the total RR charge generated by D-branes (and O-planes in orientifolds) must add up to zero in compact space. This is known as tadpole cancellation condition

$$
\sum_{a} N_{a}\left[\Pi_{a}\right]=0
$$

Stacking $N$ D-branes on top of each other open strings living on the stack will form the adjoint representation of a $\mathrm{U}(N)$ gauge group. Each intersection of two stacks of D-branes with respective gauge groups $\mathrm{U}(N)$ and $\mathrm{U}(M)$ accomodates chiral fermions in a bifundamental representation of $\mathrm{U}(N) \times \mathrm{U}(M)$. If the branes of both stacks $\Pi_{a}$ and $\Pi_{b}$ are calibrated w.r.t. the same 3 -form in (2.3) (i.e. $\theta_{a}=\theta_{b}$ ) one scalar particle at each intersection point becomes massless and fills together with the fermion a chiral $\mathcal{N}=1$ multiplet.

In intersecting D-brane models one can have also topologically non-trivial solutions to the e.o.m. of the open string, called a worldsheet instanton. Open string instantons are Riemannian surfaces embedded into the target space by placing the boundary of the surface on 1-cycles lying in the compact factor of D-brane worldvolumes. Yukawa couplings in Type IIA occur from open string instantons connecting three intersection points where, for instance, the Higgs and a left- and right handed fermion live. To fulfill the e.o.m. the string instanton must be a holomorphic disc $D$ calibrated w.r.t. $\omega$. The volume of such an instanton can be associated with the classical value of the string action and because this is minimised, the instanton volume is also minimised. The area $A$ of $D$ in target space will determine the size of the Yukawa couplings. For each triplet of intersection points there can be infinitely many instanton solutions since the Euclidean worldsheet can wrap two-cycles in compact space an arbitrary integer number of times. Therefore, the Yukawa coupling, $Y_{i j k}$, is expressed as an infinite sum over such wrapping numbers

$$
Y_{i j k} \propto \sum e^{-\frac{A_{i j k}}{2 \pi \alpha^{\prime}}}
$$

where each component in $(i, j, k)$ labels inequivalent intersections of a brane pair. So, the triplet stands for a triplet of chiral multiplets. The individual labels differ among multiplets in the same representation, i.e. they can be viewed as family indices. (Note, however, that the number of families can in general depend on the representation, unlike in the Standard Model). The area $A_{i j k}=\int_{\partial D} \omega$ is minimised, where $\partial D$ is the the boundary's postion of the holomorphic disc in the target space. The area depends on $(i, j, k)$ due to the condition that $\partial D$ has to pass through points which are equivalent to the ones labeled by $(i, j, k)$. 


\section{Branes on non-factorisable six-tori}

Explicit computations by means of comformal field theory can be performed when the Calabi-Yau 3-fold degenerates to an orbifold (on which string theory is still well defined). For simplicity, we will discuss the case of toroidal compactification. To obtain a compactification we first decompose ten dimensional spacetime into a product of four dimensional spacetime times $\mathbb{R}^{6}$. By identifying points related by lattice shifts $\mathbb{R}^{6}$ is replaced by a six-torus $T^{6}$. As an example for a non-factorisable lattice we consider the $\mathrm{SO}(12)$ root lattice, i.e. w.r.t. Cartesian coordinates on $\mathbb{R}^{6}$ the compactification lattice is generated by $\mathrm{SO}(12)$ simple roots,

$$
\begin{aligned}
& e_{1}=(1,-1,0,0,0,0), \\
& e_{2}=(0,1,-1,0,0,0), \\
& e_{3}=(0,0,1,-1,0,0), \\
& e_{4}=(0,0,0,1,-1,0), \\
& e_{5}=(0,0,0,0,1,-1), \\
& e_{6}=(0,0,0,0,1,1) .
\end{aligned}
$$

It is convenient to introduce three complex coordinates on $\mathbb{R}^{6}$, as

$$
z_{i}=x_{2 i-1}+\mathrm{i} x_{2 i}, \quad i=1,2,3
$$

So, the Kähler two-form is

$$
\omega=\frac{i}{2} \sum_{i=1}^{3} \mathrm{~d} z_{i} \wedge \mathrm{d} \bar{z}_{i} .
$$

The associated $(3,0)$-form satisfying $(2.2)$ is

$$
\Omega=d z_{1} \wedge d z_{2} \wedge d z_{3}
$$

Note, that (2.2) fixes $\Omega$ only up to a phase. This ambiguity is already parameterised by $\theta$ in the calibration form $\operatorname{Re}\left(\mathrm{e}^{-\mathrm{i} \theta} \Omega\right)$. Another ambiguity lies in the choice of complex structure, i.e. by picking the pairs of real coordinates which form a complex one in (3.2). Here, we anticipate that finally one wants to take an orbifold of $T^{6}$ where the point group consists of simultaneous rotations in the three complex planes such that the rotation angles add up to zero. Our choice ensures that $\omega$ as well as $\Omega$ are invariant under the point group. Now, we need to specify special Lagrangian submanifolds w.r.t. $\operatorname{Re}\left(\mathrm{e}^{-\mathrm{i} \theta} \Omega\right)$. Viewed as submanifolds of $\mathbb{C}^{3}$, they should have the additional property that constant shifts in the coordinates provide again special Lagrangian submanifolds. This ensures that we can compactify $\mathbb{C}^{3}$ to a $T^{6}$. The first condition in (2.3) is satisfied if the location of the D-brane can be obtained from three equations relating $z_{i}$ to its complex conjugate for each $i=1,2,3$. In particular we take the submanifold of solutions to

$$
x_{2 i}=\left(\tan \varphi_{i}\right) x_{2 i-1}, \quad i=1,2,3 .
$$


Eq. (3.5) defines angles up to shifts by $\pi$. However, we define the angles up to shifts by $2 \pi$ by encoding also the orientation of a line via the $\operatorname{sign}$ of $\cos \varphi_{i}$ and, if $\cos \varphi_{i}=0$, via the $\operatorname{sign}$ of $\sin \varphi_{i}$. The second condition in (2.3) leads to

$$
\sin \left(\theta-\varphi_{1}-\varphi_{2}-\varphi_{3}\right)=0,
$$

and thus specifies the form w.r.t. which our submanifold is calibrated. Indeed the calibration form on our submanifold is

$$
\left.\operatorname{Re}\left(\mathrm{e}^{-\mathrm{i} \theta} \Omega\right)\right|_{\Pi}=\frac{\cos \left(\theta-\varphi_{1}-\varphi_{2}-\varphi_{3}\right)}{\cos \varphi_{1} \cos \varphi_{2} \cos \varphi_{3}} d x_{1} \wedge d x_{2} \wedge d x_{3}
$$

If $\left|\varphi_{i}\right|=\pi / 2$ we replace $d x_{i}$ by $\left(\cot \varphi_{i}\right) d y_{i}$. The metric induced on the submanifold is

$$
\left.g\right|_{\Pi}=\sum_{i=1}^{3}\left(1+\tan ^{2} \varphi_{i}\right) d x_{i}^{2}
$$

leading to the volume form

$$
\left.\operatorname{vol}\right|_{\Pi}=\sqrt{\operatorname{det} g} d x_{1} \wedge d x_{2} \wedge d x_{3}=\frac{1}{\cos \varphi_{1} \cos \varphi_{2} \cos \varphi_{3}} d x_{1} \wedge d x_{2} \wedge d x_{3},
$$

where, in the second step, we have chosen the branch of the square root such that shifting individual angles by $\pi$ reverses the orientation, i.e. changes the sign of the volume form. Equating (3.9) to the expression in (3.7) removes one of the two solutions to (3.6), and yields

$$
\theta=\varphi_{1}+\varphi_{2}+\varphi_{3}
$$

Shifting $\theta$ by $\pi$ produces and extra sign in the calibration form which can be absorbed by inverting the orientation on the submanifold. A D-brane on a submanifold of one orientation is equivalent to an anti D-brane on the same submanifold with inverted orientation.

In summary, we can parametrise the 3-cycle homology class as in the factorisable case

$$
\Pi_{a}=\prod_{i=1}^{3}\left(n_{a}^{i}[a]+m_{a}^{i}[b]\right) \text { with } n_{a}^{i}, m_{a}^{i} \in \mathbb{Z}
$$

where

$$
[a]=(1,0), \quad[b]=(0,1)
$$

are one-cycles on a $T^{2}=\mathbb{R}^{2} / \Lambda$, and $\Lambda$ is a quadratic lattice of unit size. The subscript $a$ labels different cycles (to be wrapped by branes). The previously used angles can be obtained from

$$
\varphi_{i}=\arctan \frac{m^{i}}{n^{i}}, \quad \begin{aligned}
& \operatorname{sign}\left(\cos \varphi_{i}\right)=\operatorname{sign}\left(n^{i}\right) \text { if } n^{i} \neq 0, \\
& \operatorname{sign}\left(\sin \varphi_{i}\right)=\operatorname{sign}\left(m^{i}\right) \text { if } n^{i}=0,
\end{aligned}
$$

where we supressed the label $a$. Later, we will turn on geometric moduli in each plane (such that the notion of factorisable versus non-factorisable is preserved). This is done by modifying (3.12) to $(i \in\{1,2,3\})$

$$
[a]^{i}=\left(a_{1}^{i}, a_{2}^{i}\right), \quad[b]^{i}=\left(b_{1}^{i}, b_{2}^{i}\right) .
$$


The value of the angles is now computed from

$$
\varphi_{i}=\arctan \frac{n^{i} a_{2}^{i}+m^{i} b_{2}^{i}}{n^{i} a_{1}^{i}+m b_{1}^{i}}, \quad \begin{aligned}
& \operatorname{sign}\left(\cos \varphi_{i}\right)=\operatorname{sign}\left(n^{i} a_{1}^{i}+m^{i} b_{1}^{i}\right) \text { if } n^{i} a_{1}^{i}+m^{i} b_{1}^{i} \neq 0, \\
& \operatorname{sign}\left(\sin \varphi_{i}\right)=\operatorname{sign}\left(n^{i} a_{2}^{i}+m^{i} b_{2}^{i}\right) \text { if } n^{i} a_{1}^{i}+m^{i} b_{1}^{i}=0 .
\end{aligned}
$$

At first, (3.11) looks applicable only to factorisable six-tori. The product is to be understood as the cross product ${ }^{1}$ mapping a triplet of one-cycles in $T^{2}$ to a three-cycle in $T^{2} \times T^{2} \times T^{2}$. However, with some modifications this notion can also be carried over to the non-factorisable $T^{6}=\mathbb{R}^{6} / \Lambda_{\mathrm{SO}(12)}[48]{ }^{2}$ Most easily this can be seen if (we drop the brane label $a$ in the present discussion)

$$
m^{i}+n^{i}=0 \quad \bmod \quad 2
$$

for all $i=1,2,3$. In this case the cross product yields trivially a closed three-cycle on $\mathbb{R}^{6} / \Lambda_{\mathrm{SO}(12)}$. Consider now the case that for one plane, say the first, the sum of the wrapping numbers is odd, i.e.

$$
m^{1}+n^{1}=\text { odd }
$$

We claim that then the cross product

$$
\left(2 n^{1}, 2 m^{1}\right) \times\left(n^{2}, m^{2}\right) \times\left(n^{3}, m^{3}\right)
$$

yields a closed three-cycle on $\mathbb{R}^{6} / \Lambda_{\mathrm{SO}(12)}$ for any integer choice of the wrapping numbers in the second and third plane. The cross product (3.18) can be viewed as a set of points

$$
M=\left\{\left(2 n^{1} x, 2 m^{1} x, n^{2} y, m^{2} y, n^{3} z, m^{3} z\right), \quad x, y, z \in[0,1]\right\} .
$$

It remains to show that this set is an invariant subset of $\mathbb{R}^{6} / \Lambda_{\mathrm{SO}(12)}$ under shifting any of the parameters $x, y, z$ by one. For $x$ this is trivial. If $n^{2}+m^{2}$ is odd the statement can be seen also for $y \rightarrow y+1$ after a suitable reparameterisation of our set $M$. To this end we first decompose the set

$$
\begin{aligned}
M & =M_{1} \cup M_{2}, \\
M_{1} & =\left\{\left(n^{1} x, m^{1} x, n^{2} y, m^{2} y, n^{3} z, m^{3} z\right), \quad x, y, z \in[0,1]\right\}, \\
M_{2} & =\left\{\left(n^{1} x+n^{1}, m^{1} x+m^{1}, n^{2} y, m^{2} y, n^{3} z, m^{3} z\right), \quad x, y, z \in[0,1]\right\} .
\end{aligned}
$$

Since both, $n^{1}+m^{1}$ and $n^{2}+m^{2}$ are odd the six dimensional vector

$$
\vec{V}=\left(-n^{1},-m^{1}, n^{2}, m^{2}, 0,0\right)
$$

is in the $\mathrm{SO}(12)$ lattice. Shifting the position vector in $M_{2}$ by that vector and forming the union of $M_{1}$ with $M_{2}$ afterwards we obtain an equivalent parameterisation of $M$ in $\mathbb{R}^{6} / \Lambda_{\mathrm{SO}(12)}$,

$$
M=\left\{\left(n^{1} x, m^{1} x, 2 n^{2} y, 2 m^{2} y, n^{3} z, m^{3} z\right), \quad x, y, z \in[0,1]\right\},
$$

\footnotetext{
${ }^{1}$ The crossproduct of three subsets $A, B$ and $C$ of a set $M$ is a subset of the Cartesian product $M \times M \times M$ given by $A \times B \times C=\{(a, b, c) \mid a \in A, b \in B, c \in C\}$.

${ }^{2}$ In [48] a three-cycle was associated with the antisymmetrised tensor product of $\mathrm{SO}(12)$ lattice vectors. Here, we just focus on the set of points representing the cycle.
} 
in which closure under $y \rightarrow y+1$ is obvious. If $m^{3}+n^{3}$ is also odd an analogous argument shows closure under $z \rightarrow z+1$. In summary, we characterise a three-cycle on $\mathbb{R}^{6} / \Lambda_{\mathrm{SO}(12)}$ by three pairs of integer wrapping numbers $\left(n^{i}, m^{i}\right), i=1,2,3$ such that one of the following conditions hold:

- Condition (3.16) holds for $i=1,2,3$. For at least two pairs $n^{i}$ and $m^{i}$ are coprime. For the remaining pair they are either also coprime or can be written as $n^{i}=2 k$, $m^{i}=2 l$ with $k+l$ odd and $k, l$ coprime.

- Condition (3.16) does not hold for exactly one pair. The integers in that pair are coprime. One of the pairs satisfying (3.16) contains coprime integers whereas the other one consists of even numbers $(2 k, 2 l)$ with coprime integers $k, l$ and $k+l$ is odd.

- Condition (3.16) does not hold for exactly two pairs. The remaining pair consists of even numbers $(2 k, 2 l)$ with coprime integers $k, l$ and $k+l$ is odd.

Otherwise the cycle is either multiply wrapped or not closed in $\mathbb{R}^{6} / \Lambda_{\mathrm{SO}(12)}$. For instance the cycle $(2,0) \times(2,0) \times(1,0)$ corresponds to a stack of two branes wrapping $(2,0) \times$ $(1,0) \times(1,0)$, for which the last condition of our list is satisfied. On the other hand, the cycle $(1,0) \times(1,0) \times(1,0)$ is not closed and has to be replaced by e.g. $(2,0) \times(1,0) \times(1,0)$.

\section{Labelling inequivalent intersections}

In order to write down general expressions (such as in (2.5)) it is useful to label different chiral multiplets by different numbers. Since chiral multiplets are localised at intersection points of two stacks of D-branes this amounts to labelling inequivalent intersection points. Let us recall how this is done for a factorisable $T^{6}$. Consider two stacks of branes wrapping three-cycles $\Pi_{a}$ and $\Pi_{b}$, respectively. These three-cycles are specified by their wrapping numbers as in (3.11), respectively an analogous expression with subscript $a$ replaced by $b$. For the factorisable $T^{6}$ the intersection number can be written as a product of three integers (viz. the intersection numbers on the $T^{2}$ factors)

$$
I_{a b}=\prod_{i=1}^{3} I_{a b}^{(i)}, \quad \text { with } I_{a b}^{(i)}=n_{a}^{i} m_{b}^{i}-m_{a}^{i} n_{b}^{i} .
$$

Therefore, it is convenient to label the intersecction point by a triplet of integers

$$
\left(j^{(1)}, j^{(2)}, j^{(3)}\right), \quad \text { with } j^{(i)} \in\left\{0,1, \ldots,\left|I_{a b}^{(i)}\right|-1\right\} .
$$

It remains to associate a particular intersection locus to a label. For the factorisable $T^{6}$ we focus on a $T^{2}$ factor which we obtain by taking the quotient of the complex plane with respect to a square unit lattice. For the moment, we consider the case that all D-branes pass through the origin. The equation determining the intersection points is

$$
\left(\begin{array}{c}
n_{a} x \\
m_{a} x
\end{array}\right)=\left(\begin{array}{c}
n_{b} y \\
m_{b} y
\end{array}\right)+\left(\begin{array}{c}
k \\
l
\end{array}\right)
$$


where $k$ and $l$ are integers corresponding to lattice shifts. The real variables $x$ and $y$ parameterise the subsets at which the D-branes are located. One finds the following positions of intersection points

$$
\Pi_{a} \cap \Pi_{b \mid T^{2}}=\left\{\left(\begin{array}{c}
n_{a} \frac{k m_{b}-l n_{b}}{I_{a b}} \\
m_{a} \frac{k m_{b}-l n_{b}}{I_{a b}}
\end{array}\right) \mid \quad k, l \in \mathbb{Z}\right\} .
$$

If the factor $\frac{k m_{b}-l n_{b}}{I_{a b}}$ is integer the corresponding intersection point is equivalent to the origin. Since $m_{a}$ and $n_{a}$ are coprime $I_{a b}$ cannot be a non trivial divisor of both numbers. The integers $k$ and $l$ specify the position of the intersection point. The relation to its label is given by

$$
k m_{b}-\ln _{b}=i \quad \bmod \quad\left|I_{a b}\right| .
$$

Notice that since $n_{b}$ and $m_{b}$ are coprime $k$ and $l$ can be arranged such that the left hand side of (4.5) equals any of the integers in $\left[0,\left|I_{a b}\right|\right)$. For each of the $T^{2}$ factors the space of inequivalent labels is given by

$$
\frac{\mathbb{Z}}{\mathbb{Z}_{\left|I_{a b}\right|}}
$$

Combining the three $T^{2}$ factors making up the factorisable $T^{6}$ we find that labels of inequivalent intersection points are triplets taking values on the factorisable three dimensional quotient lattice

$$
\left(i^{(1)}, i^{(2)}, i^{(3)}\right) \in \bigotimes_{k=1}^{3} \frac{\mathbb{Z}}{\mathbb{Z}\left|I_{a b}^{(k)}\right|} .
$$

For the non-factorisable $T^{6}$ we will see now that inequivalent labels can take values on a non-factorisable three dimensional quotient lattice. Instead of (4.3) we have to solve the full six dimensional equation and find (up to $\mathrm{SO}(12)$ lattice shifts)

$$
\begin{array}{r}
\Pi_{a} \cap \Pi_{b}=\left\{\left(A_{a b} n_{a}^{1}, A_{a b} m_{a}^{1}, B_{a b} n_{a}^{2}, B_{a b} m_{a}^{2}, C_{a b} n_{a}^{3}, C_{a b} m_{a}^{3}\right)^{\mathrm{T}}, \quad\right. \text { with } \\
A_{a b}=\frac{t_{1} m_{b}^{1}-t_{2} n_{b}^{1}}{I_{a b}^{(1)}}, B_{a b}=\frac{t_{3} m_{b}^{2}-t_{4} n_{b}^{2}}{I_{a b}^{(2)}}, C_{a b}=\frac{t_{5} m_{b}^{3}-t_{6} n_{b}^{3}}{I_{a b}^{(3)}}, \\
\left.I_{a b}^{(i)}=\left(n_{a}^{i} m_{b}^{i}-n_{b}^{i} m_{a}^{i}\right), \quad \vec{t}=\left(t_{1}, t_{2}, t_{3}, t_{4}, t_{5}, t_{6}\right)^{\mathrm{T}} \in \Lambda_{\mathrm{SO}(12)}\right\}
\end{array}
$$

The actual space of inequivalent labels depends on which of the configurations listed in section 3 is realised. Altogether there are nine different configurations to consider which we will do in the following.

Case 1. Here, we consider the case that

$$
\forall_{i \in\{1,2,3\}} \forall_{x \in\{a, b\}}\left(n_{x}^{i}+m_{x}^{i}=0 \bmod 2, \quad \text { g.c.d. }\left(n_{x}^{i}, m_{x}^{i}\right)=1\right),
$$

where g.c.d. (...) assigns the greatest common divisor to the list of integers in its argument. The inequivalent labels are in a subset of

$$
\left\{\left(t_{1} m_{b}^{1}-t_{2} n_{b}^{1}, t_{3} m_{b}^{2}-t_{4} n_{b}^{2}, t_{5} m_{b}^{3}-t_{6} n_{b}^{3}\right) \mid \vec{t} \in \Lambda_{\mathrm{SO}(12)}\right\}=\Lambda_{\mathrm{SO}(6)},
$$


where $\Lambda_{\mathrm{SO}(6)}$ is the lattice generated by $\mathrm{SO}(6)$ simple roots,

$$
\alpha_{1}=(1,-1,0), \alpha_{2}=(0,1,-1), \alpha_{3}=(0,1,1) .
$$

Shifting the $i^{\text {th }}$ component of the three-dimensional label by an integer multiple of $I_{a b}^{(i)}$ leads to an equivalent label. Thus our quotient lattice of inequivalent labels is

$$
\frac{\Lambda_{\mathrm{SO}(6)}}{\mathbb{Z}_{I_{a b}^{(1)}} \otimes \mathbb{Z}_{I_{a b}^{(2)}} \otimes \mathbb{Z}_{I_{a b}^{(3)}}}
$$

The number of inequivalent intersection points is given by the index of the quotient lattice

$$
\left|\frac{\Lambda_{\mathrm{SO}(6)}}{\mathbb{Z}_{I_{a b}^{(1)}} \otimes \mathbb{Z}_{I_{a b}^{(2)}} \otimes \mathbb{Z}_{I_{a b}^{(3)}}}\right|=\left|\frac{E_{I_{a b}^{(1)} \otimes \mathbb{Z}_{I_{a b}^{(2)}} \otimes \mathbb{Z}_{a b}^{(3)}}}{E_{\Lambda_{\mathrm{SO}(6)}}}\right|,
$$

where the E's are the determinants of the dreibein corresponding to the lattice indicated by the subscript. Especially

$$
\begin{aligned}
E_{\mathbb{Z}_{a b}^{(1)} \otimes \mathbb{Z}_{I_{a b}^{(2)}} \otimes \mathbb{Z}_{I_{a b}^{(3)}}} & =\operatorname{det}\left(\begin{array}{ccc}
I_{a b}^{(1)} & 0 & 0 \\
0 & I_{a b}^{(2)} & 0 \\
0 & 0 & I_{a b}^{(3)}
\end{array}\right)=I_{a b}^{(1)} I_{a b}^{(2)} I_{a b}^{(3)}, \\
E_{\Lambda_{\mathrm{SO}(6)}} & =\operatorname{det}\left(\begin{array}{ccc}
1 & -1 & 0 \\
0 & 1 & -1 \\
0 & 1 & 1
\end{array}\right)=2 .
\end{aligned}
$$

Hence the number of inequivalent intersection labels equals the modulus of the intersection number $I_{a b}$, where $[47,48]$

$$
I_{a b}=\frac{1}{2} \prod_{i=1}^{3} I_{a b}^{(i)}
$$

As an aside we notice that in the case considered here all the $I_{a b}^{(i)}$ are even which can be easily seen by rewriting

$$
I_{a b}^{(i)}=\frac{n_{a}^{i}-m_{a}^{i}}{2}\left(n_{b}^{i}+m_{b}^{i}\right)-\frac{n_{a}^{i}+m_{a}^{i}}{2}\left(n_{b}^{i}-m_{b}^{i}\right),
$$

which is the difference between two even numbers. This confirms that we are taking the quotient with respect to a sublattice of $\Lambda_{\mathrm{SO}(6)}$.

Case 2. Here we consider the configuration

$$
\begin{aligned}
& \text { g.c.d. }\left(n_{a}^{1}, m_{a}^{1}\right)=2, \quad \frac{n_{a}^{1}+m_{a}^{1}}{2}=\text { odd, } \\
& \forall_{i \in\{2,3\}}\left(n_{a}^{i}+m_{a}^{i}=0 \bmod 2, \quad \text { g.c.d. }\left(n_{a}^{i}, m_{a}^{i}\right)=1\right), \\
& \forall_{i \in\{1,2,3\}}\left(n_{b}^{i}+m_{b}^{i}=0 \bmod 2, \quad \text { g.c.d. }\left(n_{b}^{i}, m_{b}^{i}\right)=1\right) .
\end{aligned}
$$

Since the configuration of stack $b$ is the same as in the previous case conclusion (4.9) still holds. Now shifting $i^{(1)}$ by an integer multiple of $I_{a b}^{(1)} / 2$ already leads to an equivalent 
point on $T^{6}$. One can show that $I_{a b}^{(1)} / 2$ can be written as the difference of an even and an odd number and hence is odd. So shifting $i^{(1)}$ by $I_{a b}^{(1)} / 2$ has to be accompanied by shifting any of the other two remaining label components by an odd number. This however leads to an inequivalent point since $I_{a b}^{(2)}$ and $I_{a b}^{(3)}$ are even. Therefore the sublattice with respect to which we take the quotient is the same as in the previous example and we arrive at the same result.

Case 3. Here, we consider the previous situation with the roles of stacks $a$ and $b$ swapped,

$$
\begin{aligned}
& \forall_{i \in\{1,2,3\}}\left(n_{a}^{i}+m_{a}^{i}=0 \bmod 2, \quad \text { g.c.d. }\left(n_{a}^{i}, m_{a}^{i}\right)=1\right), \\
& \text { g.c.d. }\left(n_{b}^{1}, m_{b}^{1}\right)=2, \quad \frac{n_{b}^{1}+m_{b}^{1}}{2}=\text { odd, } \\
& \forall_{i \in\{2,3\}}\left(n_{b}^{i}+m_{b}^{i}=0 \bmod 2, \quad \text { g.c.d. }\left(n_{b}^{i}, m_{b}^{i}\right)=1\right) .
\end{aligned}
$$

Now, the label takes values in

$$
\left\{\left(t_{1} m_{b}^{1}-t_{2} n_{b}^{1}, t_{3} m_{b}^{2}-t_{4} n_{b}^{2}, t_{5} m_{b}^{3}-t_{6} n_{b}^{3}\right) \mid \vec{t} \in \Lambda_{\mathrm{SO}(12)}\right\}=2 \mathbb{Z} \otimes \mathbb{Z} \otimes \mathbb{Z}
$$

where $2 \mathbb{Z}$ denotes the set of even numbers. The difference to (4.9) comes about as follows. Since $n_{b}^{1}$ and $m_{b}^{1}$ are even the first component of the index is even. It is not affected by shifts

$$
\left(\begin{array}{l}
t_{1} \\
t_{2}
\end{array}\right) \rightarrow\left(\begin{array}{c}
t_{1} \\
t_{2}
\end{array}\right)+\left(\begin{array}{c}
\frac{n_{b}^{1}}{2} \\
\frac{m_{b}^{1}}{2}
\end{array}\right) .
$$

This shift does not correspond to the first two components of a $\Lambda_{\mathrm{SO}(12)}$ lattice vector. So, if we shift any of the other label-components by an integer we can always associate a $\Lambda_{\mathrm{SO}(12)}$ lattice vector to it by leaving or adding the contribution (4.20). So, now we obtain for the space of inequivalent labels the quotient lattice

$$
\frac{2 \mathbb{Z} \otimes \mathbb{Z} \otimes \mathbb{Z}}{\mathbb{Z}_{I_{a b}^{(1)}} \otimes \mathbb{Z}_{I_{a b}^{(2)}} \otimes \mathbb{Z}_{I_{a b}^{(3)}}},
$$

whose index is again $\left|I_{a b}\right|$ as it should be.

Case 4. The fourth configuration we consider is

$$
\begin{aligned}
& \text { g.c.d. }\left(n_{a}^{1}, m_{a}^{1}\right)=2, \quad \frac{n_{a}^{1}+m_{a}^{1}}{2}=\text { odd, } \\
& \forall_{i \in\{2,3\}}\left(\text { g.c.d. }\left(n_{a}^{i}, m_{a}^{i}\right)=1\right), \quad n_{a}^{2}+m_{a}^{2}=\text { even, } \quad n_{a}^{3}+m_{a}^{3}=\text { odd, } \\
& \forall_{i \in\{1,2,3\}}\left(n_{b}^{i}+m_{b}^{i}=0 \bmod 2, \quad \text { g.c.d. }\left(n_{b}^{i}, m_{b}^{i}\right)=1\right) .
\end{aligned}
$$

Since the configuration of stack $b$ is the same as in the first case the space of inequivalent labels will be again some quotient of $\Lambda_{\mathrm{SO}(6)}$. This time the sublattice of equivalence shifts looks slightly different, it is generated by

$$
\beta_{1}=\left(I_{a b}^{(1)}, 0,0\right), \quad \beta_{2}=\left(0, I_{a b}^{(2)}, 0\right), \quad \beta_{3}=\left(\frac{1}{2} I_{a b}^{(1)}, 0, I_{a b}^{(3)}\right) .
$$

The determinant of this dreibein is the same as in case one and hence the index of the quotient lattice is still $\left|I_{a b}\right|$. 
Case 5. We consider the previous case with $a$ and $b$ interchanged,

$$
\begin{aligned}
& \forall_{i \in\{1,2,3\}}\left(n_{a}^{i}+m_{a}^{i}=0 \bmod 2, \quad \text { g.c.d. }\left(n_{a}^{i}, m_{a}^{i}\right)=1\right), \\
& \text { g.c.d. }\left(n_{b}^{1}, m_{b}^{1}\right)=2, \quad \frac{n_{b}^{1}+m_{b}^{1}}{2}=\text { odd, } \\
& \forall_{i \in\{2,3\}}\left(\text { g.c.d. }\left(n_{b}^{i}, m_{b}^{i}\right)=1\right), \quad n_{b}^{2}+m_{b}^{2}=\text { even, } \quad n_{b}^{3}+m_{b}^{3}=\text { odd. }
\end{aligned}
$$

The quotient lattice of inequivalent intersection labels is the same as in (4.21).

Case 6. The set of wrapping numbers satisfies

$$
\begin{aligned}
& \forall_{x \in\{a, b\}}\left(\text { g.c.d. }\left(n_{x}^{1}, m_{x}^{1}\right)=2, \quad \frac{n_{x}^{1}+m_{x}^{1}}{2}=\text { odd }\right), \\
& \forall_{x \in\{a, b\}} \forall_{i \in\{2,3\}}\left(n_{x}^{i}+m_{x}^{i}=0 \bmod 2, \quad \text { g.c.d. }\left(n_{x}^{i}, m_{x}^{i}\right)=1\right) .
\end{aligned}
$$

Inequivalent labels are in (4.21). This does not change if we permute the three pairs of wrapping numbers for stack $a$ whereas such a permutation in stack $b$ results in a corresponding permuation of the order of the factors in the numerator in (4.21).

Case 7. We consider configurations of the form

$$
\begin{aligned}
& \text { g.c.d. }\left(n_{a}^{1}, m_{a}^{1}\right)=2, \quad \frac{n_{a}^{1}+m_{a}^{1}}{2}=\text { odd, } \\
& \forall_{i \in\{2,3\}}\left(n_{a}^{i}+m_{a}^{i}=0 \bmod 2, \quad \text { g.c.d. }\left(n_{a}^{i}, m_{a}^{I}\right)=1\right), \\
& \text { g.c.d. }\left(n_{b}^{1}, m_{b}^{1}\right)=2, \quad \frac{n_{b}^{1}+m_{b}^{1}}{2}=\text { odd, } \\
& \forall_{i \in\{2,3\}}\left(\text { g.c.d. }\left(n_{b}^{i}, m_{b}^{i}\right)=1\right), \\
& n_{b}^{2}+m_{b}^{2}=0 \bmod 2, \quad n_{b}^{3}+m_{b}^{3}=\text { odd. }
\end{aligned}
$$

The space of inequivalent labels is again quotient lattice (4.21). Permuting the pairs of wrapping number in stack $a$ does not change this. Permuting the pairs of wrapping numbers in stack $b$ alters the position of $2 \mathbb{Z}$ in (4.21).

Case 8. We take the configuration of the previous case with $a$ and $b$ swapped,

$$
\begin{aligned}
& \text { g.c.d. }\left(n_{a}^{1}, m_{a}^{1}\right)=2, \quad \frac{n_{a}^{1}+m_{a}^{1}}{2}=\text { odd, } \\
& \forall_{i \in\{2,3\}}\left(\text { g.c.d. }\left(n_{a}^{i}, m_{a}^{i}\right)=1\right), \\
& n_{a}^{2}+m_{a}^{2}=0 \bmod 2, \quad n_{a}^{3}+m_{a}^{3}=\text { odd, } \\
& \text { g.c.d. }\left(n_{b}^{1}, m_{b}^{1}\right)=2, \quad \frac{n_{b}^{1}+m_{b}^{1}}{2}=\text { odd, } \\
& \forall_{i \in\{2,3\}}\left(n_{b}^{i}+m_{b}^{i}=0 \bmod 2, \quad \text { g.c.d. }\left(n_{b}^{i}, m_{b}^{I}\right)=1\right) .
\end{aligned}
$$

The space of inequivalent labels are equivalence classes in the set of lattice vectors

$$
2 \mathbb{Z} \otimes \mathbb{Z} \otimes \mathbb{Z}
$$


with respect to shifts generated by

$$
\beta_{1}=\left(I_{a b}^{(1)}, 0,0\right), \quad \beta_{2}=\left(\frac{I_{a b}^{(1)}}{2}, I_{a b}^{(2)}, 0\right), \quad \beta_{3}=\left(0,0, I_{a b}^{(3)}\right) .
$$

The index of this quotient lattice is $\left|I_{a b}\right|$. Permuting pairs of wrapping numbers gives again very similar results and will not be discussed in detail.

Case 9. Finally, we look at configurations of the form

$$
\begin{aligned}
& \text { g.c.d. }\left(n_{a}^{1}, m_{a}^{1}\right)=2, \quad \frac{n_{a}^{1}+m_{a}^{1}}{2}=\text { odd, } \\
& \forall_{i \in\{2,3\}}\left(\text { g.c.d. }\left(n_{a}^{i}, m_{a}^{i}\right)=1\right), \\
& n_{a}^{2}+m_{a}^{2}=0 \bmod 2, \quad n_{a}^{3}+m_{a}^{3}=\text { odd, } \\
& \text { g.c.d. }\left(n_{b}^{1}, m_{b}^{1}\right)=2, \quad \frac{n_{b}^{1}+m_{b}^{1}}{2}=\text { odd, } \\
& \forall_{i \in\{2,3\}}\left(\text { g.c.d. }\left(n_{b}^{i}, m_{b}^{i}\right)=1\right), \\
& n_{b}^{2}+m_{b}^{2}=0 \bmod 2, \quad n_{b}^{3}+m_{b}^{3}=\text { odd. }
\end{aligned}
$$

Here, the space of inequivalent labels is identical to the previous case 8 .

\section{$5 \quad$ Yukawa couplings}

In [31] Yukawa couplings have been computed as a sum over worldsheet instantons. These instantons are Euclidean open string worldsheets. The worldsheet parameters take values on a disc which is holomorphically or anti-holomorphically mapped to a two dimensional surface in target space. The boundary of the disc is mapped to curves lying within the wordlvolume of D6-branes and passing through three intersection points. The three multiplets, whose Yukawa coupling is being computed, are each localised at one of the three intersection points. As explained in [31] (especially in their appendix $\mathrm{A}^{3}$ ) projections of the worldsheet image onto each of the three complex planes (appearing in $T^{6}=\mathbb{C}^{3} / \Lambda_{\mathrm{SO}(12)}$ ) are straight triangles (possibly points). The instanton action is the area of the worldsheet image in units of $\alpha^{\prime}$. For a holomorphic or anti-holomorphic embedding this area is given by the sum of the areas of the three projection triangles (where a point has zero area).

Here, we will also include the possibility of brane stacks not passing through the origin, i.e. we shift the branes position by

$$
\Pi_{x} \rightarrow \Pi_{x}+\sum_{i=1}^{3} \frac{\epsilon_{x}^{i}}{\left(n_{x}^{i}\right)^{2}+\left(m_{x}^{i}\right)^{2}}\left(-m_{x}^{i} \mathbf{k}_{2 i-1}+n_{x}^{i} \mathbf{k}_{2 i}\right),
$$

where the $\mathbf{k}_{l}$ 's form the canonical basis of $\mathbb{R}^{6}$ (one in the $l^{\text {th }}$ row and zero else) and $x \in\{a, b, c\}$. The positions of intersection points are shifted by the $\epsilon$ 's. For the loci of the

\footnotetext{
${ }^{3}$ The arguments given there do not depend on the choice of compactification lattice.
} 
triangle's vertices in the $i^{\text {th }}$ plane we find ${ }^{4}$

$$
\begin{aligned}
& (a b)_{i}=\left(\begin{array}{c}
n_{b}^{i} \\
m_{b}^{i}
\end{array}\right)\left(\frac{i^{(i)}}{I_{b a}^{(i)}}+\frac{\epsilon_{a}^{i}}{I_{a b}^{(i)}}-\frac{\left(m_{b}^{i} m_{a}^{i}+n_{b}^{i} n_{a}^{i}\right) \epsilon_{b}}{\left(\left(n_{b}^{i}\right)^{2}+\left(m_{b}^{i}\right)^{2}\right) I_{a b}^{(i)}}\right)+ \\
& \left(\begin{array}{c}
-m_{b}^{i} \\
n_{b}^{i}
\end{array}\right) \frac{\epsilon_{b}^{i}}{\left(n_{b}^{i}\right)^{2}+\left(m_{b}^{i}\right)^{2}}+\left(\begin{array}{c}
p_{2 i-1} \\
p_{2 i}
\end{array}\right) \text {, } \\
& (a c)_{i}=\left(\begin{array}{c}
n_{a}^{i} \\
m_{a}^{i}
\end{array}\right)\left(\frac{j^{(i)}}{I_{a c}^{(i)}}+\frac{\epsilon_{c}^{i}}{I_{c a}^{(i)}}-\frac{\left(m_{a}^{i} m_{c}^{i}+n_{a}^{i} n_{c}^{i}\right) \epsilon_{a}}{\left(\left(n_{a}^{i}\right)^{2}+\left(m_{a}^{i}\right)^{2}\right) I_{c a}^{(i)}}\right)+ \\
& \left(\begin{array}{c}
-m_{a}^{i} \\
n_{a}^{i}
\end{array}\right) \frac{\epsilon_{a}^{i}}{\left(n_{a}^{i}\right)^{2}+\left(m_{a}^{i}\right)^{2}}+\left(\begin{array}{c}
q_{2 i-1} \\
q_{2 i}
\end{array}\right) \\
& (b c)_{i}=\left(\begin{array}{c}
n_{c}^{i} \\
m_{c}^{i}
\end{array}\right)\left(\frac{k^{(i)}}{I_{c b}^{(i)}}+\frac{\epsilon_{b}^{i}}{I_{b c}^{(i)}}-\frac{\left(m_{b}^{i} m_{c}^{i}+n_{b}^{i} n_{c}^{i}\right) \epsilon_{c}}{\left(\left(n_{c}^{i}\right)^{2}+\left(m_{c}^{i}\right)^{2}\right) I_{b c}^{(i)}}\right)+ \\
& \left(\begin{array}{c}
-m_{c}^{i} \\
n_{c}^{i}
\end{array}\right) \frac{\epsilon_{c}^{i}}{\left(n_{c}^{i}\right)^{2}+\left(m_{c}^{i}\right)^{2}}+\left(\begin{array}{c}
t_{2 i-1} \\
t_{2 i}
\end{array}\right),
\end{aligned}
$$

where $\left(i^{(1)}, i^{(2)}, i^{(3)}\right),\left(j^{(1)}, j^{(2)}, j^{(3)}\right),\left(k^{(1)}, k^{(2)}, k^{(3)}\right)$ label inequivalent intersection points between stacks $a$ and $b$, stacks $a$ and $c$, stacks $b$ and $c$, respectively. The vectors $\left(p_{1}, \ldots, p_{6}\right)$, $\left(q_{1}, \ldots, q_{6}\right)$ and $\left(t_{1}, \ldots, t_{6}\right)$ belong to the compactification lattice $\Lambda_{\mathrm{SO}(12)}$. We denote by $z_{a}^{(i)}, z_{b}^{(i)}, z_{c}^{(i)}(i \in\{1,2,3\})$ two-dimensional directional vectors connecting vertices of the triangle, specifically

$$
\begin{aligned}
& z_{a}^{(i)}=\overrightarrow{(a b)_{i}(a c)_{i}}, \\
& z_{b}^{(i)}=\overrightarrow{(b c)_{i}(a b)_{i}}, \\
& z_{c}^{(i)}=\overrightarrow{(a c)_{i}(b c)_{i}} .
\end{aligned}
$$

These vectors are parallel to the edges of the triangle and have to add up to zero if the three points (5.2) are indeed vertices of a triangle,

$$
z_{a}^{(i)}+z_{b}^{(i)}+z_{c}^{(i)}=0
$$

This condition together with the requirement that the vectors in (5.3) are parallel to projections of cycles wrapped by the stack of D-branes connecting the corresponding intersection points (projection of stack $a$ onto the $i^{\text {th }}$ plane for $z_{a}^{(i)}$ and so on) reduce the number of parameters in (5.2). Before imposing these conditions it proves useful to relabel the intersection points,

$$
i^{(i)} \rightarrow i^{(i)} I_{a c}^{(i)} / d_{a}^{(i)}, \quad j^{(i)} \rightarrow j^{(i)} I_{c b}^{(i)} / d_{c}^{(i)}, \quad k^{(i)} \rightarrow k^{(i)} I_{b a}^{(i)} / d_{b}^{(i)} .
$$

\footnotetext{
${ }^{4}$ Although many details of the calculation do not differ from the factorisable case [31] we present it for the sake of our presentation's self-containedness.
} 
where

$$
d_{a}^{(i)}=\text { g.c.d. }\left(I_{a b}^{(i)}, I_{a c}^{(i)}\right), \quad d_{b}^{(i)}=\text { g.c.d. }\left(I_{b a}^{(i)}, I_{b c}^{(i)}\right), \quad d_{c}^{(i)}=\text { g.c.d. }\left(I_{c a}^{(i)}, I_{c b}^{(i)}\right) .
$$

Let us pause to point out a difference to the factorisable $T^{6}$ where $d_{a}^{(i)}=d_{b}^{(i)}=d_{c}^{(i)}=$ $d^{(i)}$. This follows from the identity

$$
\left(\begin{array}{c}
n_{b}^{i} \\
m_{b}^{i}
\end{array}\right) I_{a c}^{(i)}=I_{a b}^{(i)}\left(\begin{array}{c}
n_{c}^{i} \\
m_{c}^{i}
\end{array}\right)+I_{b c}^{(i)}\left(\begin{array}{c}
n_{a}^{i} \\
m_{a}^{i}
\end{array}\right) .
$$

For instance, $n_{b}^{i}$ and $m_{b}^{i}$ are coprime on a factorisable $T^{6}$ and hence $I_{a c}^{(i)}$ must be divisible by g.c.d. $\left(I_{a b}^{(i)}, I_{b c}^{(i)}\right)$. Hence, the greatest common divisor of all three intersection numbers, $d^{(i)}$, equals the greatest common divisor of any pair. In the non-factorisable case, however, it can happen that e.g. g.c.d. $\left(n_{b}^{i}, m_{b}^{i}\right)=2$ in which case one would conclude $d^{(i)}=d_{b}^{(i)} / 2$. Imagine for instance that g.c.d. $\left(n_{b}^{1}, m_{b}^{1}\right)=2$ whereas $\left(n_{a}^{1}, m_{a}^{1}\right)$ and $\left(n_{c}^{1}, m_{c}^{1}\right)$ are pairs of coprime numbers. Then $I_{a b}^{(1)}$ and $I_{b c}^{(1)}$ are twice the numbers belonging to the factorisable $T^{6}$ whereas $I_{a c}^{(1)}$ does not change. So, in that case $d_{b}^{(1)}=2 d^{(1)}$.

In terms of the relabelled intersection points the directional vectors in (5.3) read

$$
\begin{aligned}
z_{a}^{(i)}= & \mathbf{v}_{a}^{i}+\left(\begin{array}{c}
n_{a}^{i} \\
m_{a}^{i}
\end{array}\right)\left(\frac{j^{(i)} I_{c b}^{(i)}}{d_{c}^{(i)} I_{a c}^{(i)}}+\frac{\epsilon_{c}^{i}}{I_{c a}^{(i)}}-\frac{\left(m_{a}^{i} m_{c}^{i}+n_{a}^{i} n_{c}^{i}\right) \epsilon_{a}^{i}}{\left(\left(n_{a}^{i}\right)^{2}+\left(m_{a}^{i}\right)^{2}\right) I_{c a}^{(i)}}\right) \\
& -\left(\begin{array}{c}
n_{b}^{i} \\
m_{b}^{i}
\end{array}\right) \frac{i^{(i)} I_{a c}^{(i)}}{d_{a}^{(i)} I_{b a}^{(i)}}+\left(\begin{array}{c}
q_{2 i-1}-p_{2 i-1} \\
q_{2 i}-p_{2 i}
\end{array}\right), \\
z_{b}^{(i)}= & \mathbf{v}_{b}^{i}+\left(\begin{array}{c}
n_{b}^{i} \\
m_{b}^{i}
\end{array}\right)\left(\frac{i^{(i)} I_{a c}^{(i)}}{d_{a}^{(i)} I_{b a}^{(i)}}+\frac{\epsilon_{a}^{i}}{I_{a b}^{(i)}}-\frac{\left(m_{b}^{i} m_{a}^{i}+n_{b}^{i} n_{a}^{i}\right) \epsilon_{b}^{i}}{\left(\left(n_{b}^{i}\right)^{2}+\left(m_{b}^{i}\right)^{2}\right) I_{a b}^{(i)}}\right) \\
& -\left(\begin{array}{c}
n_{c}^{i} \\
m_{c}^{i}
\end{array}\right) \frac{k^{(i)} I_{b a}^{(i)}}{d_{b}^{(i)} I_{c b}^{(i)}}+\left(\begin{array}{c}
p_{2 i-1}-t_{2 i-1} \\
p_{2 i}-t_{2 i}
\end{array}\right), \\
z_{c}^{(i)}= & \mathbf{v}_{c}^{i}+\left(\begin{array}{c}
n_{c}^{i} \\
m_{c}^{i}
\end{array}\right)\left(\frac{k^{(i)} I_{b a}^{(i)}}{d_{b}^{(i)} I_{c b}^{(i)}}+\frac{\epsilon_{b}^{i}}{I_{b c}^{(i)}}-\frac{\left(m_{b}^{i} m_{c}^{i}+n_{b}^{i} n_{c}^{i}\right) \epsilon_{c}^{i}}{\left(\left(n_{c}^{i}\right)^{2}+\left(m_{c}^{i}\right)^{2}\right) I_{b c}^{(i)}}\right) \\
& -\left(\begin{array}{c}
n_{a}^{i} \\
m_{a}^{i}
\end{array}\right) \frac{j^{(i)} I_{c b}^{(i)}}{d_{c}^{(i)} I_{a c}^{(i)}}+\left(\begin{array}{c}
t_{2 i-1}-q_{2 i-1} \\
t_{2 i}-q_{2 i}
\end{array}\right),
\end{aligned}
$$

with the two dimensional vectors

$$
\begin{aligned}
\mathbf{v}_{a}^{i}= & -\left(\begin{array}{c}
n_{b}^{i} \\
m_{b}^{i}
\end{array}\right)\left(\frac{\epsilon_{a}^{i}}{I_{a b}^{(i)}}-\frac{\left(m_{b}^{i} m_{a}^{i}+n_{b}^{i} n_{a}^{i}\right) \epsilon_{b}}{\left(\left(n_{b}^{i}\right)^{2}+\left(m_{b}^{i}\right)^{2}\right) I_{a b}^{(i)}}\right)+\left(\begin{array}{c}
-m_{a}^{i} \\
n_{a}^{i}
\end{array}\right) \frac{\epsilon_{a}^{i}}{\left(n_{a}^{i}\right)^{2}+\left(m_{a}^{i}\right)^{2}} \\
& -\left(\begin{array}{c}
-m_{b}^{i} \\
n_{b}^{i}
\end{array}\right) \frac{\epsilon_{b}^{i}}{\left(n_{b}^{i}\right)^{2}+\left(m_{b}^{i}\right)^{2}},
\end{aligned}
$$




$$
\begin{aligned}
\mathbf{v}_{b}^{i}= & -\left(\begin{array}{c}
n_{c}^{i} \\
m_{c}^{i}
\end{array}\right)\left(\frac{\epsilon_{b}^{i}}{I_{b c}^{(i)}}-\frac{\left(m_{b}^{i} m_{c}^{i}+n_{b}^{i} n_{c}^{i}\right) \epsilon_{c}}{\left(\left(n_{c}^{i}\right)^{2}+\left(m_{c}^{i}\right)^{2}\right) I_{b c}^{(i)}}\right)+\left(\begin{array}{c}
-m_{b}^{i} \\
n_{b}^{i}
\end{array}\right) \frac{\epsilon_{b}^{i}}{\left(n_{b}^{i}\right)^{2}+\left(m_{b}^{i}\right)^{2}} \\
& -\left(\begin{array}{c}
-m_{c}^{i} \\
n_{c}^{i}
\end{array}\right) \frac{\epsilon_{c}^{i}}{\left(n_{c}^{i}\right)^{2}+\left(m_{c}^{i}\right)^{2}}, \\
\mathbf{v}_{c}^{i}= & -\left(\begin{array}{c}
n_{a}^{i} \\
m_{a}^{i}
\end{array}\right)\left(\frac{\epsilon_{c}^{i}}{I_{c a}^{(i)}}-\frac{\left(m_{a}^{i} m_{c}^{i}+n_{a}^{i} n_{c}^{i}\right) \epsilon_{a}}{\left(\left(n_{a}^{i}\right)^{2}+\left(m_{a}^{i}\right)^{2}\right) I_{c a}^{(i)}}\right)+\left(\begin{array}{c}
-m_{c}^{i} \\
n_{c}^{i}
\end{array}\right) \frac{\epsilon_{c}^{i}}{\left(n_{c}^{i}\right)^{2}+\left(m_{c}^{i}\right)^{2}} \\
& -\left(\begin{array}{c}
-m_{a}^{i} \\
n_{a}^{i}
\end{array}\right) \frac{\epsilon_{a}^{i}}{\left(n_{a}^{i}\right)^{2}+\left(m_{a}^{i}\right)^{2}} .
\end{aligned}
$$

Conditions on the $\mathrm{SO}(12)$ lattice vectors $p, q$ and $t$ arise upon imposing that vectors in (5.8) are parallel to two dimensional projections of the cycles wrapped by the corresponding Dbranes. For instance $z_{a}^{(i)}$ should be parallel to $\left(n_{a}^{i}, m_{a}^{i}\right)^{\mathrm{T}}$. Therefore the scalar product of $z_{a}^{(i)}$ with $\left(m_{a}^{i},-n_{a}^{i}\right)^{\mathrm{T}}$ has to vanish. This yields three $(i \in\{1,2,3\})$ linear Diophantine equations

$$
n_{a}^{i}\left(q_{2 i}-p_{2 i}\right)-m_{a}^{i}\left(q_{2 i-1}-p_{2 i-1}\right)=-\frac{i^{(i)} I_{a c}^{(i)}}{d_{a}^{(i)}} .
$$

Each equation is solvable only if the r.h.s. is devisable by g.c.d. $\left(n_{a}^{(i)}, m_{a}^{(i)}\right)$. However, even for g.c.d. $\left(n_{a}^{(i)}, m_{a}^{(i)}\right)=2$ this does not impose additional conditions since in that case $i^{(i)}$ is even (see e.g. case 3 of previous section). There are infinitely many solutions,

$$
\left(\begin{array}{c}
q_{2 i-1}-p_{2 i-1} \\
q_{2 i}-p_{2 i}
\end{array}\right)=-\frac{i^{(i)}}{d_{a}^{(i)}}\left(\begin{array}{c}
n_{c}^{i} \\
m_{c}^{i}
\end{array}\right)+q_{a}^{(i)}\left(\begin{array}{c}
n_{a}^{i} \\
m_{a}^{i}
\end{array}\right)
$$

where $q_{a}^{(i)}$ have to be chosen such that $q-p$ on the l.h.s. is in $\Lambda_{\mathrm{SO}(12)}$. That is, the vector on the l.h.s. must have integer components and in addition the condition

$$
\sum_{i=1}^{3}\left(-q_{a}^{(i)}\left(n_{a}^{i}+m_{a}^{i}\right)+\frac{i^{(i)}\left(n_{c}^{i}+m_{c}^{i}\right)}{d_{a}^{(i)}}\right)=0 \bmod 2,
$$

is satisfied. Also notice that $\mathbf{v}_{a}^{i}$ drops out of the scalar product in (5.10) implying that it is parallel to $\left(n_{a}^{i}, m_{a}^{i}\right)^{\mathrm{T}}$ and hence

$$
\mathbf{v}_{a}^{i}=\left(\begin{array}{c}
n_{a}^{i} \\
m_{a}^{i}
\end{array}\right) \frac{\left\langle\mathbf{v}_{a}^{i},\left(\begin{array}{c}
n_{a}^{i} \\
m_{a}^{i}
\end{array}\right)\right\rangle}{\left(n_{a}^{i}\right)^{2}+\left(m_{a}^{i}\right)^{2}}
$$

Using this as well as the identities in (5.7) and

$$
\frac{m_{a}^{i} m_{c}^{i}+n_{a}^{i} n_{c}^{i}}{I_{a c}^{(i)}}+\frac{n_{a}^{i} n_{b}^{i}+m_{a}^{i} m_{b}^{i}}{I_{b a}^{(i)}}=\frac{\left(\left(n_{a}^{i}\right)^{2}+\left(m_{a}^{i}\right)^{2}\right) I_{b c}^{(i)}}{I_{a c}^{(i)} I_{b a}^{(i)}}
$$


one finally finds

$$
z_{a}^{(i)}=\left(\begin{array}{c}
n_{a}^{i} \\
m_{a}^{i}
\end{array}\right) I_{b c}^{(i)}\left(\frac{i^{(i)}}{d_{a}^{(i)} I_{a b}^{(i)}}+\frac{j^{(i)}}{d_{c}^{(i)} I_{c a}^{(i)}}+\frac{I_{b c}^{(i)} \epsilon_{a}^{i}+I_{c a}^{(i)} \epsilon_{b}^{i}+I_{a b}^{(i)} \epsilon_{c}^{i}}{I_{c a}^{(i)} I_{a b}^{(i)} I_{b c}^{(i)}}+\frac{q_{a}^{(i)}}{I_{b c}^{(i)}}\right) .
$$

Analogously one finds

$$
\begin{aligned}
& z_{b}^{(i)}=\left(\begin{array}{c}
n_{b}^{i} \\
m_{b}^{i}
\end{array}\right) I_{c a}^{(i)}\left(\frac{i^{(i)}}{d_{a}^{(i)} I_{a b}^{(i)}}+\frac{k^{(i)}}{d_{b}^{(i)} I_{b c}^{(i)}}+\frac{I_{b c}^{(i)} \epsilon_{a}^{i}+I_{c a}^{(i)} \epsilon_{b}^{i}+I_{a b}^{(i)} \epsilon_{c}^{i}}{I_{c a}^{(i)} I_{a b}^{(i)} I_{b c}^{(i)}}+\frac{q_{b}^{(i)}}{I_{c a}^{(i)}}\right), \\
& z_{c}^{(i)}=\left(\begin{array}{c}
n_{c}^{i} \\
m_{c}^{i}
\end{array}\right) I_{a b}^{(i)}\left(\frac{j^{(i)}}{d_{c}^{(i)} I_{c a}^{(i)}}+\frac{k^{(i)}}{d_{b}^{(i)} I_{b c}^{(i)}}+\frac{I_{b c}^{(i)} \epsilon_{a}^{i}+I_{c a}^{(i)} \epsilon_{b}^{i}+I_{a b}^{(i)} \epsilon_{c}^{i}}{I_{c a}^{(i)} I_{a b}^{(i)} I_{b c}^{(i)}}+\frac{q_{c}^{(i)}}{I_{a b}^{(i)}}\right),
\end{aligned}
$$

where the $q_{b}$ 's and $q_{c}$ 's satisfy respective conditions,

$$
\begin{aligned}
-\frac{k^{(i)}}{d_{b}^{(i)}}\left(\begin{array}{c}
n_{a}^{i} \\
m_{a}^{i}
\end{array}\right)+q_{b}^{(i)}\left(\begin{array}{c}
n_{b}^{i} \\
m_{b}^{i}
\end{array}\right) & \in \mathbb{Z}^{2}, \\
\sum_{i=1}^{3}\left(-q_{b}^{(i)}\left(n_{b}^{i}+m_{b}^{i}\right)+\frac{k^{(i)}\left(n_{a}^{i}+m_{a}^{i}\right)}{d_{b}^{(i)}}\right) & =0 \bmod 2, \\
-\frac{j^{(i)}}{d_{c}^{(i)}}\left(\begin{array}{c}
n_{b}^{i} \\
m_{b}^{i}
\end{array}\right)+q_{c}^{(i)}\left(\begin{array}{c}
n_{c}^{i} \\
m_{c}^{i}
\end{array}\right) & \in \mathbb{Z}^{2}, \\
\sum_{i=1}^{3}\left(-q_{c}^{(i)}\left(n_{c}^{i}+m_{c}^{i}\right)+\frac{j^{(i)}\left(n_{b}^{i}+m_{b}^{i}\right)}{d_{c}^{(i)}}\right) & =0 \bmod 2 .
\end{aligned}
$$

Finally, we demand the triangles to close in each plane, i.e.

$$
z_{a}^{(i)}+z_{b}^{(i)}+z_{c}^{(i)}=0, \quad \text { for } \quad i \in\{1,2,3\} .
$$

For each plane this provides two equations for three variables $q_{a}^{(i)}, q_{b}^{(i)}, q_{c}^{(i)}$. Hence there will be three free parameters $\ell^{(1)}, \ell^{(2)}, \ell^{(3)}$. The solutions are

$$
\begin{aligned}
& q_{a}^{(i)}=\frac{k^{(i)}}{d_{b}^{(i)}}+\frac{I_{b c}^{(i)} \ell^{(i)}}{d^{(i)}}, \\
& q_{b}^{(i)}=\frac{j^{(i)}}{d_{c}^{(i)}}+\frac{I_{c a}^{(i)} \ell^{(i)}}{d^{(i)}}, \\
& q_{c}^{(i)}=\frac{i^{(i)}}{d_{a}^{(i)}}+\frac{I_{a b}^{(i)} \ell^{(i)}}{d^{(i)}},
\end{aligned}
$$

where

$$
d^{(i)}=\text { g.c.d. }\left(d_{a}^{(i)}, d_{b}^{(i)}, d_{c}^{(i)}\right) .
$$

Selection rules resulting from (5.11), (5.12) and (5.18)-(5.21) impose further conditions on these parameters. We call the set of solutions $\Lambda_{3}$, i.e.

$$
\left(\ell^{(1)}, \ell^{(2)}, \ell^{(3)}\right) \in \Lambda_{3},
$$


$\Lambda_{3}$ denotes a three dimensional lattice possibly with a lable dependent off-set. The worldsheet area of an instanton coupling strings localised at intersections $i, j, k$ is (recall each label consists of three components)

$$
\begin{aligned}
A_{i, j, k}(\ell) & =\frac{1}{2} \sum_{h=1}^{3} \sqrt{\left|z_{a}^{(h)}\right|^{2}\left|z_{b}^{(h)}\right|^{2}-\left(z_{a}^{(h)^{T}} z_{b}^{(h)}\right)^{2}} \\
& =\frac{1}{2} \sum_{h=1}^{3}\left|I_{a b}^{(h)} I_{b c}^{(h)} I_{c a}^{(h)}\right|\left(\frac{i^{(h)}}{d_{a}^{(h)} I_{a b}^{(h)}}+\frac{j^{(h)}}{d_{c}^{(h)} I_{c a}^{(h)}}+\frac{k^{(h)}}{d_{b}^{(h)} I_{b c}^{(h)}}+\tilde{\epsilon}^{(h)}+\frac{\ell^{(h)}}{d^{(h)}}\right)^{2}
\end{aligned}
$$

with

$$
\tilde{\epsilon}^{(i)}=\frac{I_{b c}^{(i)} \epsilon_{a}^{i}+I_{c a}^{(i)} \epsilon_{b}^{i}+I_{a b}^{(i)} \epsilon_{c}^{i}}{I_{c a}^{(i)} I_{a b}^{(i)} I_{b c}^{(i)}}
$$

Deforming the metric in each of the complex planes does not affect the property of $T^{6}$ to be non-factorisable. This can be easily included by 'covariantising' our expression. Two dimensional scalar products, as they occur e.g. in (5.1) are modified in an obvious way. We keep $\epsilon^{(i)}$ with the understanding that its normalisation depends on the metric in the $i^{\text {th }}$ plane. Antisymmetric combinations like $I_{a b}^{(i)}$ transform as densities and should be multiplied with $\sqrt{g^{(i)}}$, where $g^{(i)}$ is the metric's determinant in the $i^{\text {th }}$ plane. It is typically replaced by the Kähler modulus

$$
g^{(i)}=(2 \pi)^{2} A^{(i)}
$$

Strictly speaking this would be only the Kähler modulus if we compactified the two components of $z^{(i)}$ on unit circles. Also in our case we expect a non-trivial $B$ field with indices in the $i^{\text {th }}$ plane to amount to a complexified $A^{(i)}$. For the deformed $2 \mathrm{~d}$ geometries we obtain

$$
A_{i, j, k}(\ell)=\frac{(2 \pi)^{2}}{2} \sum_{h=1}^{3} A^{(h)}\left|I_{a b}^{(h)} I_{b c}^{(h)} I_{c a}^{(h)}\right|\left(\frac{i^{(h)}}{d_{a}^{(h)} I_{a b}^{(h)}}+\frac{j^{(h)}}{d_{c}^{(h)} I_{c a}^{(h)}}+\frac{k^{(h)}}{d_{b}^{(h)} I_{b c}^{(h)}}+\tilde{\epsilon}^{(h)}+\frac{\ell^{(h)}}{d^{(h)}}\right)^{2}
$$

The Yukawa coupling is obtained as a sum over worldsheet instantons

$$
Y_{i j k}=h_{\mathrm{qu}} \sigma_{a b c} \sum_{\ell \in \Lambda_{3}} \exp \left(-\frac{A_{i, j, k}(\ell)}{2 \pi \alpha^{\prime}}\right)
$$

where

$$
\sigma_{a b c}=\operatorname{sign}\left(I_{a b} I_{b c} I_{c a}\right),
$$

and $h_{\mathrm{qu}}$ is a quantum contribution in accordance with the corresponding discussion in [31]. Their discussion about Wilson lines could be carried over to the present situation as well. By picking a basis in $\Lambda_{3}$ and replacing the sum over lattice vectors by a sum over its integer components our expression for the Yukawa coupling can be brought into the form of a multi-theta function as anticipated in [31]. 


\begin{tabular}{|c|c|c|c|}
\hline plane $(i)$ & 1 & 2 & 3 \\
\hline$\left(n_{a}^{i}, m_{a}^{i}\right)$ & $(2,0)$ & $(1,0)$ & $(1,0)$ \\
\hline$\left(n_{b}^{i}, m_{b}^{i}\right)$ & $(0,1)$ & $(0,2)$ & $(0,1)$ \\
\hline$\left(n_{c}^{i}, m_{c}^{i}\right)$ & $(3,1)$ & $(1,1)$ & $(1,1)$ \\
\hline$I_{a b}^{(i)}$ & 2 & 2 & 1 \\
\hline$I_{a b}$ & \multicolumn{3}{|c|}{2} \\
\hline$I_{a c}^{(i)}$ & 2 & 1 & 1 \\
\hline$I_{a c}$ & \multicolumn{3}{|c|}{1} \\
\hline$I_{b c}^{(i)}$ & -3 & -2 & 1 \\
\hline$I_{b c}$ & \multicolumn{3}{|c}{} \\
\hline
\end{tabular}

Table 1. Cycles and intersection numbers for first example.

\section{Examples}

In the present section we will look at two examples. The first example is designed to focus just on specific characteristics of branes on non-factorisable six-tori. The second example will be slightly more complex also featuring subtleties in cases where some intersection points lose their label in the process of relabelling.

First, we discuss a very simple setup where particularities due to the non-factorisable compactification can be demonstrated. We choose the wrapping numbers according to table 1.

Inequivalent labels $i$ of the $a b$-intersection take values in

$$
i \in \frac{2 \mathbb{Z} \otimes \mathbb{Z} \otimes \mathbb{Z}}{\Gamma}
$$

where $\Gamma$ is a lattice generated by

$$
(2,1,0), \quad(0,2,0), \quad(0,1,1) .
$$

There are two equivalence classes which we represent as

$$
i \in\{(0,0,0),(2,0,0)\} .
$$

For the $a c$-intersections there is only one inequivalent label which we choose as

$$
j=(0,0,0) .
$$

Finally, the label of $b c$-intersections $k$ takes values in

$$
k \in \frac{\mathbb{Z} \otimes 2 \mathbb{Z} \otimes \mathbb{Z}}{\mathbb{Z}_{3} \otimes \mathbb{Z}_{2} \otimes \mathbb{Z}}
$$

Again we can choose representatives of equivalence classes with vanishing second and third components,

$$
k \in\{(0,0,0),(1,0,0),(2,0,0)\} .
$$




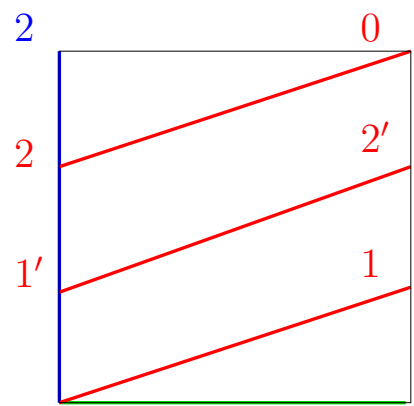

$0,0,0$ $1^{\prime}, 2^{\prime}$

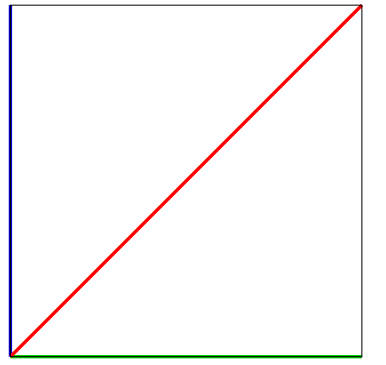

$0,0,0$

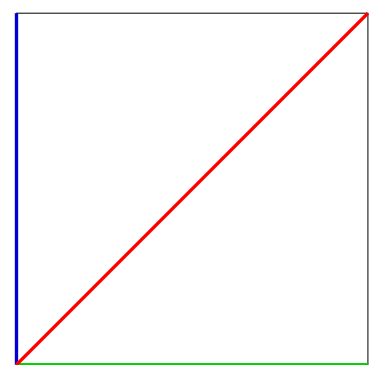

$0,0,0$

Figure 1. A simple example showing subtleties for non-factorisable tori. Since inequivalent labels can be chosen to have only non-vanishing first components we wrote just the first component. Labels are assigned according to our rules. (In the particular example, relabelling does not affect them). Values for $i, j, k$ are depicted in blue, green, red, respectively. Brane stacks $a, b, c$ are drawn in green, blue, read, respectively. Interssections with primed labels are related to intersections with the same unprimed labels by a lattice shift with non-vanishing components in the first and second plane.

Since all our labels have non-zero entries only in the first component we replace the threedimensional vectors by their first components in the following. Note further, that in our particular example relabelling according to (5.5) maps labels to equivalent labels. The assignment of labels to intersection points is visualised in figure 1. The largest Yukawa coupling is among fields which are localised at $i=0, j=0$ and $k=0$. Selection rules yield the condition

$$
\left(\begin{array}{c}
-6 \ell^{(1)} \\
0 \\
-2 \ell^{(2)} \\
0 \\
\ell^{(3)} \\
0
\end{array}\right),\left(\begin{array}{c}
0 \\
-2 \ell^{(1)} \\
0 \\
2 \ell^{(2)} \\
0 \\
\ell^{(3)}
\end{array}\right),\left(\begin{array}{c}
6 \ell^{(1)} \\
2 \ell^{(1)} \\
2 \ell^{(2)} \\
2 \ell^{(2)} \\
\ell^{(3)} \\
\ell^{(3)}
\end{array}\right) \in \Lambda_{\mathrm{SO}(12)} .
$$

They are solved by

$$
\ell^{(1)}=\frac{l^{(1)}}{2}, \quad \ell^{(2)}=\frac{l^{(2)}}{2}, \quad \ell^{(3)}=l^{(3)}, \quad l \equiv\left(\begin{array}{l}
l^{(1)} \\
l^{(2)} \\
l^{(3)}
\end{array}\right) \in \Lambda_{\mathrm{SO}(6)},
$$

where $\Lambda_{\mathrm{SO}(6)}$ consists of three dimensional vectors whose integer components sum to an even number. For the Yukawa coupling we find

$$
Y_{000}=-h_{\mathrm{qu}} \sum_{l \in \Lambda_{\mathrm{SO}(6)}} \exp \left\{-\frac{\pi}{\alpha^{\prime}}\left(3 A^{(1)}\left(l^{(1)}\right)^{2}+A^{(2)}\left(l^{(2)}\right)^{2}+A^{(3)}\left(l^{(3)}\right)^{2}\right)\right\} .
$$

It is also illustrative to compare $Y_{201}$ to $Y_{001}$ which would be identical on a factorisable $T^{6}$. In both cases the selection rules remain the same as for $Y_{000}$. The couplings can be expressed as a sum over $\mathrm{SO}(6)$ lattice vectors. Instead of writing down the full instanton 


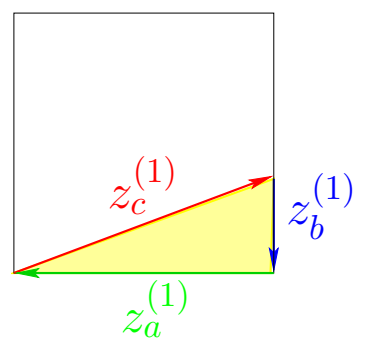

Figure 2. The triangle is the worldsheet instanton providing the leading contribution to $Y_{201}$. Shown is the projection on the first plane. The area on the other two planes is zero.

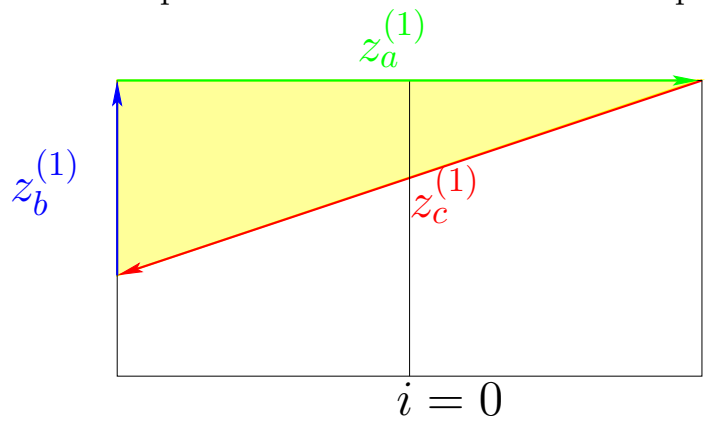

Figure 3. The triangle is the worldsheet instanton providing one of the leading contribution to $Y_{001}$. Shown is the projection on the first plane. The area on the other two planes is zero.

sum let us focus on leading contributions, i.e. smallest triangles. We start with $Y_{201}$. In this case, the smallest triangle has zero area in the second and third plane and is depicted in figure 2. This can be easily confirmed by an explicit computation with $l=0$

$$
\begin{aligned}
& z_{a}^{1}=\left(\begin{array}{l}
2 \\
0
\end{array}\right)(-3)\left(\frac{1}{2}-\frac{1}{3}\right)=\left(\begin{array}{c}
-1 \\
0
\end{array}\right), \\
& z_{b}^{1}=\left(\begin{array}{l}
0 \\
1
\end{array}\right)(-2)\left(\frac{1}{2}-\frac{1}{3}\right)=\left(\begin{array}{c}
0 \\
-\frac{1}{3}
\end{array}\right), \\
& z_{c}^{1}=\left(\begin{array}{l}
3 \\
1
\end{array}\right) 2\left(\frac{1}{2}-\frac{1}{3}\right)=\left(\begin{array}{c}
1 \\
\frac{1}{3}
\end{array}\right) .
\end{aligned}
$$

For $Y_{001}$ we find for $l=0$

$$
\begin{aligned}
& z_{a}^{1}=\left(\begin{array}{l}
2 \\
0
\end{array}\right)(-3)\left(-\frac{1}{3}\right)=\left(\begin{array}{l}
2 \\
0
\end{array}\right), \\
& z_{b}^{1}=\left(\begin{array}{l}
0 \\
1
\end{array}\right)(-2)\left(-\frac{1}{3}\right)=\left(\begin{array}{l}
0 \\
\frac{2}{3}
\end{array}\right), \\
& z_{c}^{1}=\left(\begin{array}{l}
3 \\
1
\end{array}\right) 2\left(-\frac{1}{3}\right)=\left(\begin{array}{l}
-2 \\
-\frac{2}{3}
\end{array}\right) .
\end{aligned}
$$

The corresponding triangle is drawn in figure 3 . In this case there is another competing 

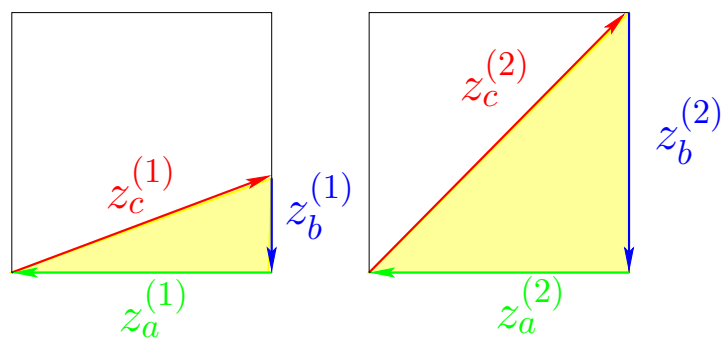

Figure 4. The triangle is the worldsheet instanton providing another leading contribution to $Y_{001}$. Shown is the projection on the first and second planes. The area on the third plane is zero. There is also a worldsheet instanton with the role of second and third plane interchanged.

contribution to the coupling. It corresponds to the choice

$$
l=\left(\begin{array}{c}
-1 \\
1 \\
0
\end{array}\right) \rightarrow \ell=\left(\begin{array}{c}
-\frac{1}{2} \\
\frac{1}{2} \\
0
\end{array}\right) .
$$

In the first plane this mimics replacing $i=0$ by $i=2$ and we obtain the same set of vectors as in (6.10)-(6.12). For the second plane we get

$$
\begin{aligned}
& z_{a}^{(2)}=\left(\begin{array}{l}
1 \\
0
\end{array}\right)(-2)\left(\frac{1}{2}\right)=\left(\begin{array}{c}
-1 \\
0
\end{array}\right), \\
& z_{b}^{(2)}=\left(\begin{array}{l}
0 \\
2
\end{array}\right)(-1)\left(\frac{1}{2}\right)=\left(\begin{array}{c}
0 \\
-1
\end{array}\right), \\
& z_{c}^{(2)}=\left(\begin{array}{l}
1 \\
1
\end{array}\right) 2\left(\frac{1}{2}\right)=\left(\begin{array}{l}
1 \\
1
\end{array}\right) .
\end{aligned}
$$

So, for $\ell$ as in (6.16) the worldsheet instanton action is given by the sum of triangles in figure 4. Depending on metric moduli this area can be smaller than the $\ell=0$ contribution in figure 3.

As a second example we look at a more generic setup where intersection points lose their label by relabelling. (A simpler discussion relevant for factorisable lattices can be found in appendix A.) Wrapping and intersection numbers are displayed in table 2.

Next, we need to label intersection points. First we will follow the prescreption (5.2). Let us begin with intersections of stacks $a$ and $b$. Intersection points $\mathbf{p}_{a b}$ are up to $\mathrm{SO}(12)$ lattice shifts given by

$$
\mathbf{p}_{a b}=\left\{\left(\frac{-3 t_{1}-t_{2}}{-3}, 0,2 \frac{t_{3}-t_{4}}{2}, 0, \frac{t_{5}-t_{6}}{3},-2 \frac{t_{5}-t_{6}}{3}\right) \mid \vec{t} \in \Lambda_{\mathrm{SO}(12)}\right\}
$$

We label those points by a triplet

$$
i=\left(-3 t_{1}-t_{2}, t_{3}-t_{4}, t_{5}-t_{6}\right),
$$

which is a lattice vector in $\Lambda_{\mathrm{SO}(6)}$. Shifting labels as

$$
i \rightarrow i+(0,2,0), \quad i \rightarrow i+(3,1,0), \quad i \rightarrow i+(0,1,3) .
$$




\begin{tabular}{|c|c|c|c|}
\hline plane $(i)$ & 1 & 2 & 3 \\
\hline$\left(n_{a}^{i}, m_{a}^{i}\right)$ & $(1,-3)$ & $(1,1)$ & $(1,1)$ \\
\hline$\left(n_{b}^{i}, m_{b}^{i}\right)$ & $(1,0)$ & $(2,0)$ & $(1,-2)$ \\
\hline$\left(n_{c}^{i}, m_{c}^{i}\right)$ & $(2,3)$ & $(4,6)$ & $(1,-1 r)$ \\
\hline$I_{a b}^{(i)}$ & 3 & -2 & -3 \\
\hline$I_{a b}$ & \multicolumn{3}{|c|}{9} \\
\hline$I_{a c}^{(i)}$ & 9 & 2 & -2 \\
\hline$I_{a c}$ & \multicolumn{3}{|c|}{-18} \\
\hline$I_{b c}^{(i)}$ & 3 & 12 & 1 \\
\hline$I_{b c}$ & \multicolumn{3}{|c}{18} \\
\hline
\end{tabular}

Table 2. Cycles and intersection numbers for second example.

leads to equivalent intersection points on $T^{6}$. Therefore, inequivalent labels belong to the lattice quotient

$$
i \in \frac{\Lambda_{\mathrm{SO}(6)}}{\Gamma_{a b}},
$$

with

$$
\Gamma_{a b}=\left\{\sum_{i=1}^{3} n_{i} \vec{e}_{i} \mid n_{i} \in \mathbb{Z}, \vec{e}_{1}=\left(\begin{array}{l}
0 \\
2 \\
0
\end{array}\right), \vec{e}_{2}=\left(\begin{array}{l}
3 \\
1 \\
0
\end{array}\right), \vec{e}_{3}=\left(\begin{array}{l}
0 \\
1 \\
3
\end{array}\right)\right\} .
$$

Relabelling according to (5.5) corresponds to

$$
\left(i^{(1)}, i^{(2)}, i^{(3)}\right) \rightarrow\left(3 i^{(1)}, i^{(2)},-2 i^{(3)}\right),
$$

where new labels are again defined up to shifts in $\Gamma_{a b}$. If new labels obtained from inequivalent labels become equivalent on the lattice quotient we do not assign a label to one of the corresponding intersection points. For instance the label $(1,0,1)$ is mapped to $(3,0,-2)$. Adding the $\Gamma_{a b}$ lattice vector $(-3,0,3)$ assigns equivalently the label $(0,0,1)$ which is however already used for the non equivalent intersection point erstwhile labelled by $(0,1,1)$. Therefore points labelled originally by $(1,0,1)$ lose their label. Analogously one finds the old label $(2,1,1)$ would be also relabelled to $(0,0,1)$. New labels for $(2,0,2)$ and $(1,1,2)$ are equivalent to label $(0,0,2)$ reserved for the relabelled $(0,0,2)$. Old labels $(2,0,0)$ and $(1,1,0)$ are mapped to $(0,0,0)$ which is already taken by the relabelled $(0,0,0)$. In table 3 the labels before and after relabelling and the corresponding coordinates on the torus are listed, a hyphen means "label lost".

Next, we investigate intersections of stacks $a$ and $c$. Up to $\mathrm{SO}(12)$ lattice shifts, intersection points are in the set

$$
\mathbf{p}_{a c}=\left\{\left(\frac{3 t_{1}-2 t_{2}}{9},-3 \frac{3 t_{1}-2 t_{2}}{9}, \frac{6 t_{3}-4 t_{4}}{2}, \frac{6 t_{3}-4 t_{4}}{2}, \frac{t_{5}+t_{6}}{2}, \frac{t_{5}+t_{6}}{2}\right) \mid \vec{t} \in \Lambda_{\mathrm{SO}(12)}\right\} .
$$




\begin{tabular}{|l|c|l|}
\hline old label $i$ & new label $i^{\prime}$ & coordinates \\
\hline$(0,0,0)$ & $(0,0,0)$ & $(0,0,0,0,0,0)$ \\
$(1,0,1)$ & - & $\left(-\frac{1}{3}, 0,0,0, \frac{1}{3},-\frac{2}{3}\right)$ \\
$(2,0,0)$ & - & $\left(-\frac{2}{3}, 0,0,0,0,0\right)$ \\
$(0,0,2)$ & $(0,0,2)$ & $\left(0,0,0,0, \frac{2}{3},-\frac{4}{3}\right)$ \\
$(2,0,2)$ & - & $\left(-\frac{2}{3}, 0,0,0, \frac{2}{3},-\frac{4}{3}\right)$ \\
$(1,1,0)$ & - & $\left(-\frac{1}{3}, 0,1,0,0,0\right)$ \\
$(0,1,1)$ & $(0,0,1)$ & $\left(0,0,1,0, \frac{1}{3},-\frac{2}{3}\right)$ \\
$(2,1,1)$ & - & $\left(-\frac{2}{3}, 0,1,0, \frac{1}{3},-\frac{2}{3}\right)$ \\
$(1,1,2)$ & - & $\left(-\frac{1}{3}, 0,1,0, \frac{1}{3},-\frac{4}{3}\right)$ \\
\hline
\end{tabular}

Table 3. Labels and coordinates of intersection points.

We see that the labels $j=\left(3 t_{1}-2 t_{2}, 6 t_{3}-4 t_{4},-t_{5}-t_{6}\right)$ are a subset of the factorised lattice $\mathbb{Z} \otimes 2 \mathbb{Z} \otimes \mathbb{Z}$ and shifting labels by

$$
j \rightarrow j+(9,0,0), \quad j \rightarrow j+(0,2,0), \quad j \rightarrow j+(0,0,2),
$$

leaves $\mathbf{p}_{a c}$ invariant on the torus. The rule for relabelling components of $j$ is given by

$$
j^{(1)} \rightarrow-j^{(1)} \bmod 9, \quad j^{(2)} \rightarrow-6 j^{(2)} \bmod 2, \quad j^{(3)} \rightarrow-j^{(3)} \bmod 2 .
$$

In table 4 one can find the coordinates of labels $j$.

Finally we need to know which labels to assign to intersections of stacks $b$ and $c$. Up to lattice shifts intersection points $\mathbf{p}_{b c}$ are given by

$$
\mathbf{p}_{b c}=\left\{\left(2 \frac{t_{2}}{3}, 3 \frac{t_{2}}{3}, 4 \frac{2 t_{4}}{12}, 6 \frac{2 t_{4}}{12}, 2 t_{5}+t_{6},-2 t_{5}-t_{6}\right) \mid \vec{t} \in \Lambda_{\mathrm{SO}(12)}\right\} .
$$

One can see that labels $k=\left(t_{2}, 2 t_{4}, 2 t_{5}+t_{6}\right)$ take values on the lattice $\mathbb{Z} \otimes 2 \mathbb{Z} \otimes \mathbb{Z}$ and intersections points are equivalent on the torus if related by the following shifts,

$$
k \rightarrow k+(0,0,1), \quad k \rightarrow k+(0,12,0), \quad k \rightarrow k+(3,6,0) .
$$

The set of inequivalent labels is represented by

$$
k \in \frac{\mathbb{Z} \otimes 2 \mathbb{Z} \otimes \mathbb{Z}}{\Gamma_{b c}},
$$

with

$$
\Gamma_{b c}=\left\{\sum_{i=1}^{3} n_{i} \vec{e}_{i} \mid n_{i} \in \mathbb{Z}, \vec{e}_{1}=\left(\begin{array}{l}
0 \\
0 \\
1
\end{array}\right), \vec{e}_{2}=\left(\begin{array}{c}
0 \\
12 \\
0
\end{array}\right), \vec{e}_{3}=\left(\begin{array}{l}
3 \\
6 \\
0
\end{array}\right)\right\} .
$$

Relabelling according to (5.5) amounts to redefining

$$
\left(k^{(1)}, k^{(2)}, k^{(3)}\right) \rightarrow\left(-k^{(1)}, k^{(2)}, 3 k^{(3)}\right) \quad \bmod \text { shifts by } \Gamma_{b c} \text { lattice vectors. }
$$

The situation is summarised in table 5 . 


\begin{tabular}{|l|l|l|}
\hline old label $j$ & new label $j^{\prime}$ & coordinates \\
\hline$(0,0,0)$ & $(0,0,0)$ & $(0,0,0,0,0,0)$ \\
$(1,0,0)$ & $(8,0,0)$ & $\left(\frac{1}{9},-\frac{1}{3}, 0,0,0,0\right)$ \\
$(2,0,0)$ & $(7,0,0)$ & $\left(\frac{2}{9},-\frac{2}{3}, 0,0,0,0\right)$ \\
$(3,0,0)$ & $(6,0,0)$ & $\left(\frac{1}{3},-1,0,0,0,0\right)$ \\
$(4,0,0)$ & $(5,0,0)$ & $\left(\frac{4}{9},-\frac{4}{3}, 0,0,0,0\right)$ \\
$(5,0,0)$ & $(4,0,0)$ & $\left(\frac{5}{9},-\frac{5}{3}, 0,0,0,0\right)$ \\
$(6,0,0)$ & $(3,0,0)$ & $\left(\frac{2}{3},-2,0,0,0,0\right)$ \\
$(7,0,0)$ & $(2,0,0)$ & $\left(\frac{7}{9},-\frac{7}{3}, 0,0,0,0\right)$ \\
$(8,0,0)$ & $(1,0,0)$ & $\left(\frac{8}{9},-\frac{8}{3}, 0,0,0,0\right)$ \\
$(0,0,1)$ & $(0,0,1)$ & $\left(0,0,0,0, \frac{1}{2}, \frac{1}{2}\right)$ \\
$(1,0,1)$ & $(8,0,1)$ & $\left(\frac{1}{9},-\frac{1}{3}, 0,0, \frac{1}{2}, \frac{1}{2}\right)$ \\
$(2,0,1)$ & $(7,0,1)$ & $\left(\frac{2}{9},-\frac{2}{3}, 0,0, \frac{1}{2}, \frac{1}{2}\right)$ \\
$(3,0,1)$ & $(6,0,1)$ & $\left(\frac{1}{3},-1,0,0, \frac{1}{2}, \frac{1}{2}\right)$ \\
$(4,0,1)$ & $(5,0,1)$ & $\left(\frac{4}{9},-\frac{4}{3}, 0,0, \frac{1}{2}, \frac{1}{2}\right)$ \\
$(5,0,1)$ & $(4,0,1)$ & $\left(\frac{5}{9},-\frac{5}{3}, 0,0, \frac{1}{2}, \frac{1}{2}\right)$ \\
$(6,0,1)$ & $(3,0,1)$ & $\left(\frac{2}{3},-2,0,0, \frac{1}{2}, \frac{1}{2}\right)$ \\
$(7,0,1)$ & $(2,0,1)$ & $\left(\frac{7}{9},-\frac{7}{3}, 0,0, \frac{1}{2}, \frac{1}{2}\right)$ \\
$(8,0,1)$ & $(1,0,1)$ & $\left(\frac{8}{9},-\frac{8}{3}, 0,0, \frac{1}{2}, \frac{1}{2}\right)$ \\
\hline
\end{tabular}

Table 4. Labels and coordinates of intersection points.

The data given in the tables 3,4 and 5 contain every information we need to compute all trilinear couplings. Our example is designed such that not all couplings differ from zero. To illustrate that we study selection rules for couplings to fields belonging to intersection label $i=(0,0,0)$. These were discussed in the previous section in (5.11), (5.12), (5.18)(5.21). For our example they take the form

$$
\left(\begin{array}{c}
q_{a}^{(1)} \\
-3 q_{a}^{(1)} \\
q_{a}^{(2)} \\
q_{a}^{(2)} \\
q_{a}^{(3)} \\
q_{a}^{(3)}
\end{array}\right),\left(\begin{array}{c}
q_{b}^{(1)}-\frac{k^{(1)}}{3} \\
k^{(1)} \\
2 q_{b}^{(2)}-\frac{k^{(2)}}{2} \\
-\frac{k^{(2)}}{2} \\
q_{b}^{(3)}-k^{(3)} \\
-2 q_{b}^{(3)}-k^{(3)}
\end{array}\right),\left(\begin{array}{c}
2 q_{c}^{(1)}-\frac{j^{(1)}}{3} \\
3 q_{c}^{(1)} \\
4 q_{c}^{(2)}-j^{(2)} \\
6 q_{c}^{(2)} \\
q_{c}^{(3)}-j^{(3)} \\
-q_{c}^{(3)}+2 j^{(3)}
\end{array}\right) \in \Lambda_{\mathrm{SO}(12)}
$$

On the other hand conditions for triangles to close fix the $q$ 's to be the form (5.23)-(5.25) which for our example read

$$
\begin{array}{rlrl}
q_{a}^{(1)} & =\frac{k^{(1)}}{3}+\ell^{(1)}, & q_{a}^{(2)}=\frac{k^{(2)}}{2}+6 \ell^{(2)}, & q_{a}^{(3)}=k^{(3)}+\ell^{(3)}, \\
q_{b}^{(1)}=\frac{j^{(1)}}{3}-3 \ell^{(1)}, & q_{b}^{(2)}=\frac{j^{(2)}}{2}-\ell^{(2)}, & q_{b}^{(3)}=j^{(3)}+2 \ell^{(3)}, \\
q_{c}^{(1)}=\ell^{(1)}, & q_{c}^{(2)}=-\ell^{(2)}, & q_{c}^{(3)}=-3 \ell^{(3)} .
\end{array}
$$




\begin{tabular}{|l|l|l|}
\hline old label $k$ & new label $k^{\prime}$ & coordinates \\
\hline$(0,0,0)$ & $(0,0,0)$ & $(0,0,0,0,0,0)$ \\
$(1,0,0)$ & $(-1,0,0)$ & $\left(-\frac{2}{3},-1,0,0,0,0\right)$ \\
$(2,0,0)$ & $(-2,0,0)$ & $\left(-\frac{4}{3},-2,0,0,0,0\right)$ \\
$(0,2,0)$ & $(0,2,0)$ & $\left(0,0,-\frac{2}{3},-1,0,0\right)$ \\
$(1,2,0)$ & $(-1,2,0)$ & $\left(-\frac{2}{3},-1,-\frac{2}{3},-1,0,0\right)$ \\
$(2,2,0)$ & $(2,2,0)$ & $\left(-\frac{4}{3},-2,-\frac{2}{3},-1,0,0\right)$ \\
$(0,4,0)$ & $(0,4,0)$ & $\left(0,0,-\frac{4}{3},-2,0,0\right)$ \\
$(1,4,0)$ & $(-1,4,0)$ & $\left(-\frac{2}{3},-1,-\frac{4}{3},-2,0,0\right)$ \\
$(2,4,0)$ & $(-2,14,0)$ & $\left(-\frac{4}{3},-2,-\frac{4}{3},-2,0,0\right)$ \\
$(0,6,0)$ & $(0,6,0)$ & $(0,0,-2,-3,0,0)$ \\
$(1,6,0)$ & $(-1,6,0)$ & $\left(-\frac{2}{3},-1,-2,-3,0,0\right)$ \\
$(2,6,0)$ & $(-2,6,0)$ & $\left(-\frac{4}{3},-2,-2,-3,0,0\right)$ \\
$(0,8,0)$ & $(0,8,0)$ & $\left(0,0,-\frac{8}{3},-4,0,0\right)$ \\
$(1,8,0)$ & $(-1,8,0)$ & $\left(-\frac{2}{3},-1,-\frac{8}{3},-4,0,0\right)$ \\
$(2,8,0)$ & $(-2,8,0)$ & $\left(-\frac{4}{3},-2,-\frac{8}{3},-4,0,0\right)$ \\
$(0,10,0)$ & $(0,10,0)$ & $\left(0,0,-\frac{10}{3},-5,0,0\right)$ \\
$(1,10,0)$ & $(-1,10,0)$ & $\left(-\frac{2}{3},-1,-\frac{10}{3},-5,0,0\right)$ \\
$(2,10,0)$ & $(-2,10,0)$ & $\left(-\frac{4}{3},-2,-\frac{10}{3},-5,0,0\right)$ \\
\hline
\end{tabular}

Table 5. Labels and coordinates of intersection points.

Plugging that into (6.32) yields conditions on the other labels, $k$ and $j$, as well as on the $\ell^{(i)}$ 's. Imposing the necessary condition on vectors in (6.32) to have integer components results in

$$
\begin{array}{llrl}
\ell^{(1)} & =-\frac{k^{(1)}}{3}+l^{(1)}, & & \text { with } l^{(1)} \in \mathbb{Z}, \\
\frac{j^{(1)}}{3}=p+\frac{k^{(1)}}{3}, & & \text { with } p \in \mathbb{Z}, \\
\ell^{(2)}=\frac{l^{(2)}}{2} & & \text { with } l^{(2)} \in \mathbb{Z}, \\
\ell^{(3)}=l^{(3)} & & \text { with } l^{(3)} \in \mathbb{Z} .
\end{array}
$$

Note that the second of the above conditions contains a restriction on possible values for labels $j$ and $k$. Hence, it is really a selection rule for non vanishing couplings. Imposing now the full condition (6.32) yields in addition

$$
l^{(1)}+l^{(2)}+p+j^{(3)}=0 \quad \bmod \quad 2 .
$$

So, up to some offset depending on $j^{(3)}+p$, the instanton sum will be a sum over the three dimensional lattice $\Lambda_{\mathrm{SO}(4)} \otimes \mathbb{Z}$, where the $\Lambda_{\mathrm{SO}(4)}$ consists of four dimensional vectors with integer components whose sum is even. 


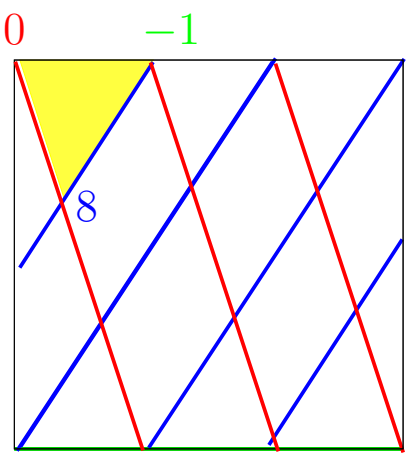

$0,8,-1$
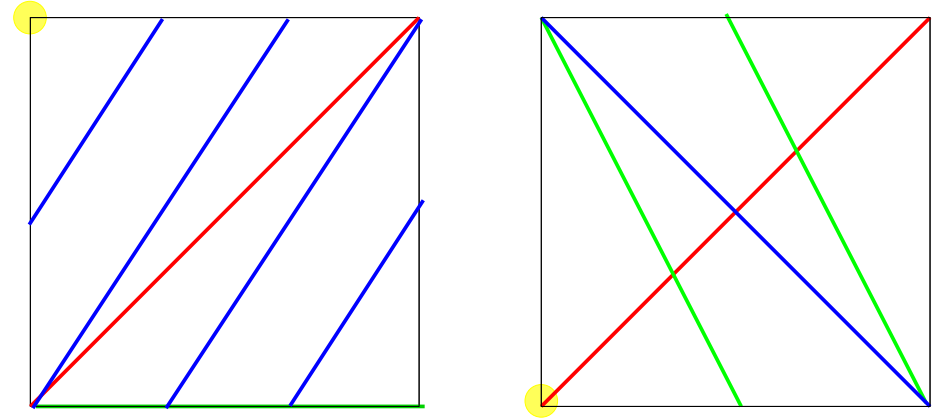

$0,8,-1$

Figure 5. The values in red, blue and green are first components of labels $i, j$ and $k$. The red, green and blue coloured lines correspond to the cycles of the branes $a, b$ and $c$. The yellow area highlighted shows the volume of the instanton with the winding number $l=(1,0,0)$ connecting intersections with labels $i=(0,0,0), j=(8,0,0)$ and $k=(-1,0,0)$ (after relabelling).

As an example we present the coupling between fields lokcalised at points corresponding to "new" labels $i=(0,0,0), j=(8,0,0)$ and $k=(-1,0,0)$,

$$
-h_{\mathrm{qu}} \sum_{l \in(1,0,0)+\Lambda_{\mathrm{S}(4)} \otimes \mathbb{Z}} \exp \left(-\frac{\pi}{\alpha^{\prime}}\left[9 A^{(1)}\left(8+3 l^{(1)}\right)^{2}+12 A^{(2)} l^{(2)^{2}}+6 A^{(3)} l^{(3)^{2}}\right]\right),
$$

where $l=\left(l^{(1)}, l^{(2)}, l^{(3)}\right)$ is the worldsheet instanton winding number. The edges of the area, spread by the instanton, are given by the vectors

$$
\left(\begin{array}{c}
z_{a}^{(1)} \\
z_{a}^{(2)} \\
z_{a}^{(3)}
\end{array}\right)=\left(\begin{array}{c}
-\frac{8}{9}+l^{(1)} \\
\frac{8}{3}-3 l^{(1)} \\
3 l^{(2)} \\
3 l^{(2)} \\
l^{(3)} \\
l^{(3)}
\end{array}\right), \quad\left(\begin{array}{c}
z_{b}^{(1)} \\
z_{b}^{(2)} \\
z_{b}^{(3)}
\end{array}\right)=\left(\begin{array}{c}
2 \frac{2}{3}-3 l^{(1)} \\
0 \\
-l^{(2)} \\
0 \\
2 l^{(3)} \\
-4 l^{(3)}
\end{array}\right), \quad\left(\begin{array}{c}
z_{c}^{(1)} \\
z_{c}^{(2)} \\
z_{c}^{(3)}
\end{array}\right)=\left(\begin{array}{c}
-1 \frac{7}{9}+2 l^{(1)} \\
-\frac{8}{3}+3 l^{(1)} \\
-2 l^{(2)} \\
-3 l^{(2)} \\
-3 \ell^{(3)} \\
3 l^{(3)}
\end{array}\right)
$$

The leading contribution to the Yukawa coupling comes either from the instanton with the winding numbers $l=(1,0,0)$ or $l=(0,1,0)$ depending on the values for $A^{(1)}$ and $A^{(2)}$. In figure 5 the worldsheet instanton with the winding number $l=(1,0,0)$ is depicted.

As we have seen, intersections sometimes lose their label in the process of relabelling. Still, we can compute Yukawa couplings involving fields localised at such intersection. The general strategy is as follows. First, consider the intersection which loses its label in the process of relabelling. We shift the old label by a fraction of an equivalence shift. The fraction is determined by the greatest common divisor of the three intersection numbers. The shifted label should be mapped to an existing new label by relabelling. The other labels need in general also to be shifted by the same fraction of corresponding equivalence shifts. Which particular equivalence shift should be taken is determined by the requirements that the shifted label exists, and its relabelled version exists. Further, associated coordinate shifts should coincide on $\mathbb{R}^{6} / \Lambda_{\mathrm{SO}(12)}$ for all three intersections. If this way selection rules 

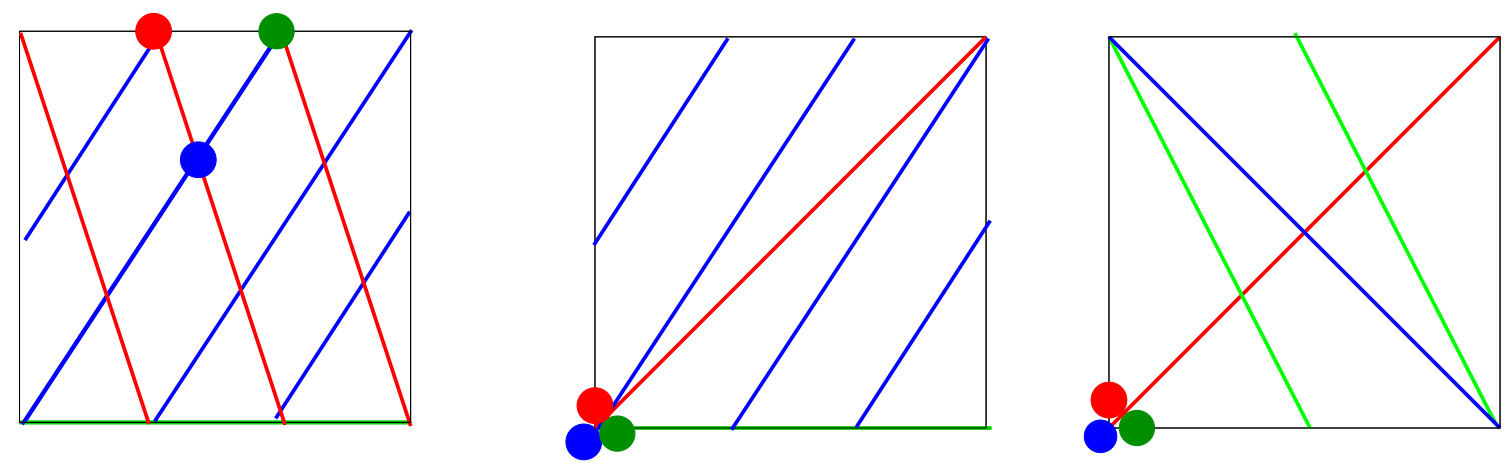

Figure 6. The intersection marked by the red disc loses its label in the process of relabelling. The Yukawa coupling to fields localised at intersections marked by blue and green discs can still be computed and is identical to the previously considered coupling.

cannot be satisfied the corresponding Yukawa coupling is zero. We illustrate the general prescription at an example.

Consider three intersections with original labels $i=(2,0,0), j=(4,0,0)$ and $k=$ $(2,6,0)$. We indicate the positions of the corresponding intersections in figure 6 . The intersection point of stacks $a$ and $b$ loses its label in the process of relabelling. Using our general prescription we change labels by third fractions of equivalence shifts, explicitly given by

$$
i \rightarrow i-\frac{1}{3}(6,0,0), \quad j \rightarrow j-\frac{1}{3}(9,0,0), \quad k \rightarrow k-\frac{1}{3}(3,18,0) .
$$

The shifted labels are identical to the original labels of our previously computed coupling. As expected, we obtain the same coupling, now.

\section{Conclusions}

The major subject of the current paper is the computation of Yukawa couplings in intersecting brane models on non-factorisable tori. The result can be found in eq. (5.31). For the factorisable torus a similar expression is given in [31]. The most significant difference is that the latter can be written as a product of three theta functions whereas (5.31) contains a multi-theta function. For our calculation we represent D-branes in a way closely related to branes on factorisable $T^{6}$. We carefully address the question of how to label intersection points. It turns out that a label in general takes values on a three dimensional quotient lattice. Our original prescription of assigning labels to intersections depends only on the two branes involved but is asymmetric under permuting them. For the computation of Yukawa couplings it is convenient to re-assign new labels depending now even on the third brane. After such relabelling the computation of Yukawa couplings leads to Diophantine equations of a particular form. They are equivalent to equations arrising in the problem of finding for a given brane a partner brane such that their intersection number takes a given value. Moreover, branes and intersection numbers correspond to ones already given by the setup. Therefore the general solution can be given in terms of wrapping numbers 
of the model and quantities reflecting the fact that parallel branes have zero intersection number. The number of these additional quantities is further reduced by more concistency conditions and one is left with three dimensional lattice vectors. These can be viewed as labelling worldsheet instantons, i.e. they can be understood as generalised wrapping numbers for open string worldsheet instantons. If these wrapping numbers take values on a factorisable lattice the Yukawa coupling will be expressed in terms of a product of three theta functions. Generically this is not possible.

We discuss subtleties for intersection numbers having non-trivial common divisors. We find that our way of relabelling intersections is not always bijective in such cases, i.e. some intersections lose their label. On the other hand there is a degeneracy in Yukawa couplings, i.e. different cubic interactions have identical coupling constants. This fact allows to relate couplings for fields with no label to others which can be computed.

We hope our work will contribute to efforts in intersecting brane model building. It should help to extend existing strategies to the study of non-factorisable tori. Recent attempts in that direction have been reported in [51]. There, $\mathbb{Z}_{12}$ orientifolds with chiral spectra are investigated. It will be interesting to find out whether techniques developed in our paper can also be used in that context. Certainly, our methods are easily adopted to all orientifolds whose point group can be also realised as an automorphism of a factorisable six-torus.

\section{Acknowledgments}

This work was supported by the SFB-Transregio TR33 "The Dark Universe" (Deutsche Forschungsgemeinschaft) and by "Bonn-Cologne Graduate School for Physics and Astronomy" (BCGS).

\section{A $\quad T^{2}$ with non-coprime intersection numbers}

To deal with the case of non-coprime intersection numbers the authors of [31] used a well motivated general ansatz together with a case by case study to compute Yukawa couplings. The second step is best performed by drawing a picture and fitting it with the general ansatz. Here, we propose an alternative treatment allowing for a more formalised deduction of the same results. That will involve relabelling intersection points in such a way that some inequivalent labels are lost. Yukawa couplings containing corresponding fields are equal to other Yukawa couplings. These contain fields belonging to intersection points retained after relabelling. Since we are considering just one $T^{2}$ factor we drop the index labelling the three complex planes. Further we consider the unit square lattice and the case that all branes pass through the origin. The analog of equations (5.2) reads

$$
\begin{aligned}
& (a b)=\left(\begin{array}{c}
n_{b} \\
m_{b}
\end{array}\right) \frac{i}{I_{b a}}+\left(\begin{array}{c}
p_{1} \\
p_{2}
\end{array}\right), \\
& (a c)=\left(\begin{array}{c}
n_{a} \\
m_{a}
\end{array}\right) \frac{j}{I_{a c}}+\left(\begin{array}{c}
q_{1} \\
q_{2}
\end{array}\right),
\end{aligned}
$$




$$
(b c)=\left(\begin{array}{c}
n_{c} \\
m_{c}
\end{array}\right) \frac{k}{I_{c b}}+\left(\begin{array}{c}
t_{1} \\
t_{2}
\end{array}\right),
$$

where now the $p_{\alpha}, q_{\alpha}, t_{\alpha}$ are just integers with no further constraints. The labels $i, j$, $k$ are defined up to shifts by integer multiples of $I_{b a}, I_{a c}$ and $I_{b c}$, respectively. Then, as explained in section 5 the greatest common divisor for any pair of intersection numbers is equal to

$$
d=\text { g.c.d. }\left(I_{a b}, I_{a c}, I_{b c}\right) .
$$

The relabelling in (5.5) simplifies to

$$
i \rightarrow i I_{a c} / d, \quad j \rightarrow j I_{c b} / d, \quad k \rightarrow k I_{b a} / d
$$

yielding

$$
\begin{aligned}
(a b) & =\left(\begin{array}{c}
n_{b} \\
m_{b}
\end{array}\right) \frac{i I_{a c}}{d I_{b a}}+\left(\begin{array}{c}
p_{1} \\
p_{2}
\end{array}\right), \\
(a c) & =\left(\begin{array}{c}
n_{a} \\
m_{a}
\end{array}\right) \frac{j I_{c b}}{d I_{a c}}+\left(\begin{array}{c}
q_{1} \\
q_{2}
\end{array}\right), \\
(b c) & =\left(\begin{array}{c}
n_{c} \\
m_{c}
\end{array}\right) \frac{k I_{b a}}{d I_{c b}}+\left(\begin{array}{c}
t_{1} \\
t_{2}
\end{array}\right) .
\end{aligned}
$$

In general it will happen that this expression misses some intersection points if e.g. $I_{a c} / d$ is devisable by $I_{a b}$. We proceed by computing directional vectors connecting intersection points

$$
\begin{aligned}
& z_{a}=\overrightarrow{(a b)(a c)}=\left(\begin{array}{c}
n_{a} \\
m_{a}
\end{array}\right) \frac{j I_{c b}}{d I_{a c}}-\left(\begin{array}{c}
n_{b} \\
m_{b}
\end{array}\right) \frac{i I_{a c}}{d I_{b a}}+\left(\begin{array}{c}
q_{1}-p_{1} \\
q_{2}-p_{2}
\end{array}\right), \\
& z_{b}=\overrightarrow{(b c)(a b)}=\left(\begin{array}{c}
n_{b} \\
m_{b}
\end{array}\right) \frac{i I_{a c}}{d I_{b a}}-\left(\begin{array}{c}
n_{c} \\
m_{c}
\end{array}\right) \frac{k I_{b a}}{d I_{c b}}+\left(\begin{array}{c}
p_{1}-t_{1} \\
p_{2}-t_{2}
\end{array}\right), \\
& z_{c}=\overrightarrow{(a c)(b c)}=\left(\begin{array}{c}
n_{c} \\
m_{c}
\end{array}\right) \frac{k I_{b a}}{d I_{c b}}-\left(\begin{array}{c}
n_{a} \\
m_{a}
\end{array}\right) \frac{j I_{c b}}{d I_{a c}}+\left(\begin{array}{c}
t_{1}-q_{1} \\
t_{2}-q_{2}
\end{array}\right) .
\end{aligned}
$$

Imposing $z_{x}$ to be parallel to $\left(n_{x}, m_{x}\right)^{\mathrm{T}}$ for $x \in\{a, b, c\}$ gives linear Diophantine equations with solutions

$$
\begin{aligned}
\left(\begin{array}{c}
q_{1}-p_{1} \\
q_{2}-p_{2}
\end{array}\right) & =\left(\begin{array}{c}
n_{a} \\
m_{a}
\end{array}\right) q_{a}-\left(\begin{array}{c}
n_{c} \\
m_{c}
\end{array}\right) \frac{i}{d}, \\
\left(\begin{array}{c}
p_{1}-t_{1} \\
p_{2}-t_{2}
\end{array}\right) & =\left(\begin{array}{c}
n_{b} \\
m_{b}
\end{array}\right) q_{b}-\left(\begin{array}{c}
n_{a} \\
m_{a}
\end{array}\right) \frac{k}{d}, \\
\left(\begin{array}{c}
t_{1}-q_{1} \\
t_{2}-q_{2}
\end{array}\right) & =\left(\begin{array}{c}
n_{c} \\
m_{c}
\end{array}\right) q_{c}-\left(\begin{array}{c}
n_{b} \\
m_{b}
\end{array}\right) \frac{j}{d},
\end{aligned}
$$


where $q_{a}, q_{b}$ and $q_{c}$ are parameters which are related via the condition for the triangle to close

$$
z_{a}+z_{b}+z_{c}=0
$$

These provide two equations for the three parameters $q_{x}$. We parameterise the solution by $l_{0}+l$ where $l_{0}$ denotes a fractional part and $l$ an integer. For the $q_{x}$ one finds

$$
q_{a}=\frac{k}{d}+\frac{\left(l_{0}+l\right) I_{b c}}{d}, \quad q_{b}=\frac{j}{d}+\frac{\left(l_{0}+l\right) I_{c a}}{d}, \quad q_{c}=\frac{i}{d}+\frac{\left(l_{0}+l\right) I_{a b}}{d} .
$$

Then the $z_{x}$ can be written as

$$
z_{a}=\left(\begin{array}{c}
n_{a} \\
m_{a}
\end{array}\right) \frac{I_{b c}}{d}\left(x_{0}+l\right), \quad z_{b}=\left(\begin{array}{c}
n_{b} \\
m_{b}
\end{array}\right) \frac{I_{c a}}{d}\left(x_{0}+l\right), \quad z_{c}=\left(\begin{array}{c}
n_{c} \\
m_{c}
\end{array}\right) \frac{I_{a b}}{d}\left(x_{0}+l\right),
$$

with

$$
x_{0}=\frac{i}{I_{a b}}+\frac{j}{I_{c a}}+\frac{k}{I_{b c}}+l_{0},
$$

which is defined up to integer shifts. One still has to take into account that the vectors on the left hand sides of (A.6) have integer entries. This provides selection rules on possible Yukawa couplings. The integer parameter $l$ contributes only integer numbers to the right hand sides and just drops off the selection rules. For $l_{0}$ running through $\frac{1}{d}, \frac{2}{d}, \ldots, \frac{d-1}{d}$ one will get different selection rules on the intersection labels. Thus $l_{0}$, indeed, represents a contribution to $x_{0}$ of the form $s(i, j, k) / d$ as postulated in [31].

To illustrate our general discussion we revisit an example discussed in [31]. The wrapping numbers are

$$
\left(n_{a}, m_{a}\right)=(1,0), \quad\left(n_{b}, m_{b}\right)=(1,2), \quad\left(n_{c}, m_{c}\right)=(1,-4) .
$$

The setup is depicted in figure 7. Here labels are allocated to intersection points according to (A.1). Relabelling as in (A.3) amounts to the replacements

$$
i:(0,1) \rightarrow(0, \text { no label }), \quad j:(0,1,2,3) \rightarrow(0,3,2,1), \quad k:(0,1,2,3,4,5) \rightarrow(0,5,4,3,2,1),
$$

where we notice that the intersection point previously labelled by $i=1$ does not have a label any more. However, for any triangle containing a vertex labelled by $i=1$ one can find a congruent triangle with vertex labelled by $i=0$. The selection rules (taken from (A.6)) imply that $k+j$ has to be even, always. For $l_{0}=0, k$ and $j$ must be even whereas for $l_{0}=\frac{1}{2}$ they must be odd. The situation can be summarised in the selection rule

$$
k+j=0 \quad \bmod \quad 2,
$$

together with (recall $x_{0}$ is defined modulo one)

$$
x_{0}=\frac{j}{4}-\frac{k}{6}+\frac{j}{2}=\frac{3 j}{4}-\frac{k}{6} .
$$

This agrees with the result reported in [31] as long as $i=0$. Now suppose, we want to obtain the Yukawa coupling for the triplet $(i, j, k)=(1,1,4)$ in figure 7 . Since the intersection point labelled by $i=1$ loses its label in the process of relabelling we first perform a shift

$$
(i, j, k) \rightarrow(i, j, k)-\frac{1}{d}\left(I_{b a}, I_{a c}, I_{c b}\right)
$$




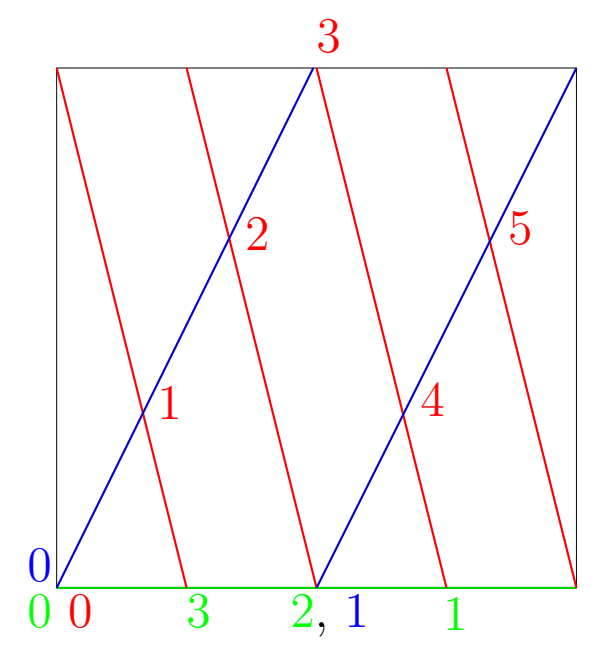

Figure 7. Setup from [31]. Labels are assigned according to our rules before relabelling. Values for $i, j, k$ are depicted in blue, green, red, respectively. Brane stacks $a, b, c$ are drawn in green, blue, read, respectively.

i.e. by $(1,2,-3)$. This tells us that the Yukawa coupling of fields localised at $(0,3,1)$ is the same. Relabelling maps this finally to $(i, j, k)=(0,1,5)$. With (A.13) and (A.9) we obtain for $l=0$

$$
x_{0}=-\frac{1}{12}, \quad z_{a}=\left(\begin{array}{c}
\frac{1}{4} \\
0
\end{array}\right), \quad z_{b}=\left(\begin{array}{c}
-\frac{1}{6} \\
-\frac{1}{3}
\end{array}\right), \quad z_{c}=\left(\begin{array}{r}
-\frac{1}{12} \\
\frac{1}{3}
\end{array}\right),
$$

in accordance with figure 7 .

Open Access. This article is distributed under the terms of the Creative Commons Attribution License (CC-BY 4.0), which permits any use, distribution and reproduction in any medium, provided the original author(s) and source are credited.

\section{References}

[1] M. Berkooz, M.R. Douglas and R.G. Leigh, Branes intersecting at angles, Nucl. Phys. B 480 (1996) 265 [hep-th/9606139] [INSPIRE].

[2] R. Blumenhagen, L. Görlich and B. Körs, Supersymmetric 4-D orientifolds of type IIA with D6-branes at angles, JHEP 01 (2000) 040 [hep-th/9912204] [INSPIRE].

[3] S. Förste, G. Honecker and R. Schreyer, Supersymmetric $\mathbb{Z}_{N} \times \mathbb{Z}_{M}$ orientifolds in 4-D with D-branes at angles, Nucl. Phys. B 593 (2001) 127 [hep-th/0008250] [INSPIRE].

[4] M. Cvetič, G. Shiu and A.M. Uranga, Three family supersymmetric standardlike models from intersecting brane worlds, Phys. Rev. Lett. 87 (2001) 201801 [hep-th/0107143] [INSPIRE].

[5] M. Cvetič, G. Shiu and A.M. Uranga, Chiral four-dimensional $N=1$ supersymmetric type $2 A$ orientifolds from intersecting D6 branes, Nucl. Phys. B 615 (2001) 3 [hep-th/0107166] [INSPIRE].

[6] R. Blumenhagen, L. Görlich and T. Ott, Supersymmetric intersecting branes on the type $2 \mathrm{~A}$ $T^{6} / \mathbb{Z}_{4}$ orientifold, JHEP 01 (2003) 021 [hep-th/0211059] [INSPIRE]. 
[7] M. Cvetič, I. Papadimitriou and G. Shiu, Supersymmetric three family SU(5) grand unified models from type IIA orientifolds with intersecting D6-branes, Nucl. Phys. B 659 (2003) 193 [Erratum ibid. B 696 (2004) 298-298] [hep-th/0212177] [INSPIRE].

[8] G. Honecker, Chiral supersymmetric models on an orientifold of $\mathbb{Z}_{4} \times \mathbb{Z}_{2}$ with intersecting D6-branes, Nucl. Phys. B 666 (2003) 175 [hep-th/0303015] [INSPIRE].

[9] M. Cvetič and I. Papadimitriou, More supersymmetric standard-like models from intersecting D6-branes on type IIA orientifolds, Phys. Rev. D 67 (2003) 126006 [hep-th/0303197] [INSPIRE].

[10] R. Blumenhagen, D. Lüst and S. Stieberger, Gauge unification in supersymmetric intersecting brane worlds, JHEP 07 (2003) 036 [hep-th/0305146] [INSPIRE].

[11] M. Cvetič, T. Li and T. Liu, Supersymmetric Pati-Salam models from intersecting D6-branes: A road to the standard model, Nucl. Phys. B 698 (2004) 163 [hep-th/0403061] [INSPIRE].

[12] G. Honecker and T. Ott, Getting just the supersymmetric standard model at intersecting branes on the $\mathbb{Z}_{6}$ orientifold, Phys. Rev. D 70 (2004) 126010 [Erratum ibid. D $7 \mathbf{1}$ (2005) 069902] [hep-th/0404055] [INSPIRE].

[13] G. Honecker, Chiral $N=14 D$ orientifolds with D-branes at angles, Mod. Phys. Lett. A 19 (2004) 1863 [hep-th/0407181] [INSPIRE].

[14] R. Blumenhagen, F. Gmeiner, G. Honecker, D. Lüst and T. Weigand, The Statistics of supersymmetric D-brane models, Nucl. Phys. B 713 (2005) 83 [hep-th/0411173] [INSPIRE].

[15] R. Blumenhagen, M. Cvetič, F. Marchesano and G. Shiu, Chiral D-brane models with frozen open string moduli, JHEP 03 (2005) 050 [hep-th/0502095] [INSPIRE].

[16] F. Gmeiner, R. Blumenhagen, G. Honecker, D. Lüst and T. Weigand, One in a billion: MSSM-like D-brane statistics, JHEP 01 (2006) 004 [hep-th/0510170] [INSPIRE].

[17] D. Bailin and A. Love, Towards the supersymmetric standard model from intersecting D6-branes on the Z-primE 6 orientifold, Nucl. Phys. B 755 (2006) 79 [Erratum ibid. B 783 (2007) 176-178] [hep-th/0603172] [INSPIRE].

[18] D. Bailin and A. Love, Almost the supersymmetric standard model from intersecting D6-branes on the $Z_{6}^{\prime}$ orientifold, Phys. Lett. B 651 (2007) 324 [Erratum ibid. B 658 (2008) 292-294] [arXiv:0705.0646] [INSPIRE].

[19] D. Bailin and A. Love, Constructing the supersymmetric Standard Model from intersecting D6-branes on the $Z_{6}^{\prime}$ orientifold, Nucl. Phys. B 809 (2009) 64 [arXiv:0801.3385] [InSPIRE].

[20] F. Gmeiner and G. Honecker, Millions of Standard Models on Z'6?, JHEP 07 (2008) 052 [arXiv:0806.3039] [INSPIRE].

[21] S. Förste and G. Honecker, Rigid D6-branes on $T^{6} /\left(\mathbb{Z}_{2} \times \mathbb{Z}_{2 M} \times \Omega R\right)$ with discrete torsion, JHEP 01 (2011) 091 [arXiv: 1010.6070] [INSPIRE].

[22] D. Bailin and A. Love, Stabilising the supersymmetric Standard Model on the $\mathbb{Z}_{6}^{\prime}$ orientifold, Nucl. Phys. B 854 (2012) 700 [arXiv:1104.3522] [INSPIRE].

[23] G. Honecker, M. Ripka and W. Staessens, The Importance of Being Rigid: D6-Brane Model Building on $T^{6} / \mathbb{Z}_{2} \times \mathbb{Z}_{6}^{\prime}$ with Discrete Torsion, Nucl. Phys. B 868 (2013) 156 [arXiv: 1209.3010] [INSPIRE].

[24] G. Honecker and W. Staessens, To Tilt or Not To Tilt: Discrete Gauge Symmetries in Global Intersecting D-brane Models, JHEP 10 (2013) 146 [arXiv:1303.4415] [INSPIRE]. 
[25] J. Ecker, G. Honecker and W. Staessens, Rigour and rigidity: Systematics on particle physics D6-brane models on $\mathbb{Z}_{2} \times \mathbb{Z}_{6}$, Fortsch. Phys. 62 (2014) 981 [arXiv:1409.1236] [InSPIRE].

[26] C. Angelantonj and A. Sagnotti, Open strings, Phys. Rept. 371 (2002) 1 [Erratum ibid. 376 (2003) 339-405] [hep-th/0204089] [INSPIRE].

[27] R. Blumenhagen, M. Cvetič, P. Langacker and G. Shiu, Toward realistic intersecting D-brane models, Ann. Rev. Nucl. Part. Sci. 55 (2005) 71 [hep-th/0502005] [inSPIRE].

[28] R. Blumenhagen, B. Körs, D. Lüst and S. Stieberger, Four-dimensional String Compactifications with D-branes, Orientifolds and Fluxes, Phys. Rept. 445 (2007) 1 [hep-th/0610327] [INSPIRE].

[29] L.E. Ibáñez and A.M. Uranga, String theory and particle physics: An introduction to string phenomenology, Cambridge University Press, Cambridge U.K. (2012).

[30] G. Aldazabal, S. Franco, L.E. Ibáñez, R. Rabadán and A.M. Uranga, Intersecting brane worlds, JHEP 02 (2001) 047 [hep-ph/0011132] [INSPIRE].

[31] D. Cremades, L.E. Ibáñez and F. Marchesano, Yukawa couplings in intersecting D-brane models, JHEP 07 (2003) 038 [hep-th/0302105] [INSPIRE].

[32] D. Cremades, L.E. Ibáñez and F. Marchesano, Computing Yukawa couplings from magnetized extra dimensions, JHEP 05 (2004) 079 [hep-th/0404229] [inSPIRE].

[33] S.A. Abel and A.W. Owen, CP violation and CKM predictions from discrete torsion, Nucl. Phys. B 651 (2003) 191 [hep-th/0205031] [INSPIRE].

[34] M. Cvetič and I. Papadimitriou, Conformal field theory couplings for intersecting D-branes on orientifolds, Phys. Rev. D 68 (2003) 046001 [Erratum ibid. D 70 (2004) 029903] [hep-th/0303083] [INSPIRE].

[35] S.A. Abel, M. Masip and J. Santiago, Flavor changing neutral currents in intersecting brane models, JHEP 04 (2003) 057 [hep-ph/0303087] [INSPIRE].

[36] S.A. Abel and A.W. Owen, Interactions in intersecting brane models, Nucl. Phys. B 663 (2003) 197 [hep-th/0303124] [inSPIRE].

[37] S.A. Abel and A.W. Owen, $N$ point amplitudes in intersecting brane models, Nucl. Phys. B 682 (2004) 183 [hep-th/0310257] [INSPIRE].

[38] D. Lüst, P. Mayr, R. Richter and S. Stieberger, Scattering of gauge, matter and moduli fields from intersecting branes, Nucl. Phys. B 696 (2004) 205 [hep-th/0404134] [INSPIRE].

[39] S.A. Abel and B.W. Schofield, One-loop Yukawas on intersecting branes, JHEP 06 (2005) 072 [hep-th/0412206] [inSPIRE].

[40] S.A. Abel and M.D. Goodsell, Intersecting brane worlds at one loop, JHEP 02 (2006) 049 [hep-th/0512072] [INSPIRE].

[41] S.A. Abel and M.D. Goodsell, Realistic Yukawa Couplings through Instantons in Intersecting Brane Worlds, JHEP 10 (2007) 034 [hep-th/0612110] [INSPIRE].

[42] D. Duo, R. Russo and S. Sciuto, New twist field couplings from the partition function for multiply wrapped D-branes, JHEP 12 (2007) 042 [arXiv:0709.1805] [INSPIRE].

[43] R. Russo and S. Sciuto, The twisted open string partition function and Yukawa couplings, JHEP 04 (2007) 030 [hep-th/0701292] [INSPIRE]. 
[44] I. Pesando, Green functions and twist correlators for $N$ branes at angles, Nucl. Phys. B 866 (2013) 87 [arXiv: 1206.1431] [INSPIRE].

[45] I. Pesando, Correlators of arbitrary untwisted operators and excited twist operators for $N$ branes at angles, Nucl. Phys. B 886 (2014) 243 [arXiv:1401.6797] [INSPIRE].

[46] I. Pesando, Canonical quantization of a string describing $N$ branes at angles, Nucl. Phys. B 889 (2014) 120 [arXiv: 1407.4627] [InSPIRE].

[47] R. Blumenhagen, J.P. Conlon and K. Suruliz, Type IIA orientifolds on general supersymmetric $Z(N)$ orbifolds, JHEP 07 (2004) 022 [hep-th/0404254] [INSPIRE].

[48] S. Förste, C. Timirgaziu and I. Zavala, Orientifold's Landscape: Non-Factorisable Six-Tori, JHEP 10 (2007) 025 [arXiv:0707.0747] [INSPIRE].

[49] T. Kimura, M. Ohta and K.-J. Takahashi, Type IIA orientifolds and orbifolds on non-factorizable tori, Nucl. Phys. B 798 (2008) 89 [arXiv:0712.2281] [INSPIRE].

[50] S. Förste and I. Zavala, Oddness from Rigidness, JHEP 07 (2008) 086 [arXiv:0806.2328] [INSPIRE].

[51] D. Bailin and A. Love, Intersecting D6-branes on the $\mathbb{Z}_{12}$-II orientifold, JHEP 01 (2014) 009 [arXiv: 1310.8215] [INSPIRE].

[52] D.D. Joyce, Riemannian Holonomy Groups and Calibrated Geometry, Oxford Graduate Texts in Mathematics, Oxford University Press, Oxford U.K. (2007).

[53] R. Harvey and H.B. Lawson Jr., Calibrated geometries, Acta Math. 148 (1982) 47.

[54] M.R. Douglas, Topics in D geometry, Class. Quant. Grav. 17 (2000) 1057 [hep-th/9910170] [INSPIRE]. 